\title{
Recent Progress in Gamow Shell Model Calculations of Drip Line Nuclei
}

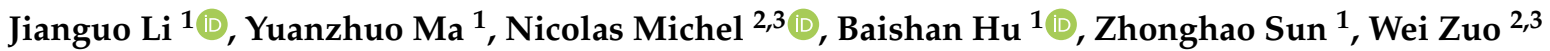 \\ and Furong $X u^{1, * \mathbb{D}}$ \\ 1 State Key Laboratory of Nuclear Physics and Technology, School of Physics, Peking University, \\ Beijing 100871, China; jianguo_li@pku.edu.cn (J.L.); yuanzhuoma@pku.edu.cn (Y.M.); \\ hubsh@pku.edu.cn (B.H.); sunzhg07@tennessee.edu (Z.S.) \\ 2 Institute of Modern Physics, Chinese Academy of Sciences, Lanzhou 730000, China; \\ nicolas.michel@impcas.ac.cn (N.M.); zuowei@impcas.ac.cn (W.Z.) \\ 3 School of Nuclear Science and Technology, University of Chinese Academy of Sciences, Beijing 100049, China \\ * Correspondence: frxu@pku.edu.cn
}

\section{check for} updates

Citation: Li, J.; Ma, Y.; Michel, N.; $\mathrm{Hu}$, B.; Sun, Z.; Zuo, W.; Xu, F. Recent Progress in Gamow Shell Model Calculations of Drip Line Nuclei. Physics 2021, 3, 977-997. https:// doi.org/10.3390/physics3040062

Received: 17 August 2021

Accepted: 25 October 2021

Published: 8 November 2021

Publisher's Note: MDPI stays neutral with regard to jurisdictional claims in published maps and institutional affiliations.

Copyright: (c) 2021 by the authors. Licensee MDPI, Basel, Switzerland. This article is an open access article distributed under the terms and conditions of the Creative Commons Attribution (CC BY) license (https:// creativecommons.org/licenses/by/ $4.0 /)$.

\begin{abstract}
The Gamow shell model (GSM) is a powerful method for the description of the exotic properties of drip line nuclei. Internucleon correlations are included via a configuration interaction framework. Continuum coupling is directly included at basis level by using the Berggren basis, in which, bound, resonance, and continuum single-particle states are treated on an equal footing in the complex momentum plane. Two different types of Gamow shell models have been developed: its first embodiment is that of the GSM defined with phenomenological nuclear interactions, whereas the GSM using realistic nuclear interactions, called the realistic Gamow shell model, was introduced later. The present review focuses on the recent applications of the GSM to drip line nuclei.
\end{abstract}

Keywords: Gamow shell model; realistic nuclear forces; phenomenological interactions; resonance; continuum; drip line nuclei

\section{Introduction}

Exotic nuclei have been studied for many years using a new generation of accelerators, which are now able to reach nuclear drip lines [1-4]. Contrary to well-bound nuclei, which are closed quantum systems, drip line nuclei can be seen as open quantum systems, as they are either weakly bound or unbound with respect to the particle emission threshold [5]. Many interesting phenomena appear at drip lines, such as a halo structure $[1,6,7]$ and particle emission in resonance states [4,8]. Continuum coupling plays an important role in these loosely bound and unbound nuclear systems [5]. The proper description of nuclei at drip lines is one of the main challenges of nuclear theory, which was mostly developed to account for the structure of well-bound nuclei $[5,6]$.

A clear consequence of the strong intertwinings of the continuum degrees of freedom and internucleon correlations at drip lines consists of the odd-even staggering found in the helium chain $[9,10]$. Indeed, odd helium isotopes (except ${ }^{3} \mathrm{He}$ ) are all resonances and bear widths of several hundreds of keV [10-12]. Conversely, the even-even helium isotopes ${ }^{4,6,8} \mathrm{He}$ are bound, with ${ }^{6,8} \mathrm{He}$ both exhibiting halo properties [13-15]. To accurately reproduce nuclear halos, many-body wave functions in asymptotic regions must be treated properly, which demands to take into account continuum coupling [1,6,7,16-19]. Adding to that, these weakly bound and unbound drip line nuclei also provide good laboratories to understand the single-particle structure, continuum coupling, internucleon correlations, and nucleon-nucleon (NN) interactions, which are not well understood in these regions.

Most present nuclear models, such as the no-core shell model (NCSM) [20], selfconsistent Green's function [21], coupled-cluster (CC) [22,23], in-medium similarity renormalization group (IM-SRG) [24], and standard shell model (SM) [25,26] have been developed for the study of well-bound nuclei, whereby continuum coupling is absent. Only few 
models explicitly include continuum coupling. The main models including both internucleon correlations and continuum coupling in a unified picture are the no-core Gamow shell model (NCGSM) [27-29], the no-core shell model with continuum (NCSMC) [30], the complex CC [31,32], the Gamow IMSRG (Gamow-IMSRG) [33], continuum shell model (CSM) [34,35], and the Gamow shell model (GSM) [19,36-39], which are extensions of the NCSM, CC, IM-SRG, and SM, respectively. However, due to their huge model space dimensions, the NCGSM and NCSMC can only be used to describe light nuclei $[27,28,30]$. Furthermore, only nuclei in the vicinity of closed-shell nuclear systems can be investigated by the complex-CC and Gamow-IMSRG methods [31-33]. CSM [34,35] takes into account the continuum effect by projecting the model space onto the subspaces of bound and scattering states in a real-energy basis, in which, resonance states are not included. Within GSM, continuum coupling is treated at basis level by way of the Berggren basis [36-38]. The latter comprises bound, resonance, and continuum scattering states, with all of these states treated on an equal footing within the Berggren ensemble [40]. Internucleon correlations in GSM are induced by configuration mixing, similarly to conventional SM. GSM has been seen to successfully reproduce many situations of physical interest [5,38]; for example, the resonances of oxygen drip line nuclei $[38,41,42]$ and the neutron halo structure of ${ }^{31} \mathrm{~F}[18]$.

The GSM was introduced in nuclear physics in 2002 [36,37], where only simple phenomenological nuclear potentials were used, while calculations were limited to only two valence neutrons outside of the inner core. After that, the GSM was extended to many valence particle systems, such as ${ }^{8} \mathrm{He}$ [17] and $p s d$-shell nuclei [43]. The realistic Gamow shell model was proposed in Refs. [44,45], with which, two- or three-particle systems could be investigated. An effective Hamiltonian based on realistic interactions was constructed by using the degenerate $\hat{Q}$-box approach; however, folded diagrams are neglected [45]. A folded diagram sums up the subset of diagrams to infinite order so as to include high-order effects. In 2017, we developed the realistic GSM method with the full $\hat{Q}$-box foldeddiagram method using the nondegenerate Berggren basis [38]. We applied it to the case of the neutron-rich oxygen isotopes up to the neutron drip line. After that, many extensions of the realistic GSM were developed, such as performing the realistic GSM in the Gamow Hartree-Fock basis (GHF) [41].

In the present review, the framework of the two types of GSM (realistic GSM and phenomenological GSM) is first introduced in Section 2. Then, we review our recent applications of GSM, including the calculations of neutron-rich oxygen and fluorine isotopes [38,39,41,42,46], neutron-rich calcium isotopes [47], and proton decays in ${ }^{16} \mathrm{Ne}$ and ${ }^{18} \mathrm{Mg}$ [48]. Finally, a short summary of the review and the future challenges of the next GSM calculations are given.

\section{Method}

GSM is built within a configuration interaction framework based on the one-body Berggren basis [5,36-38]. The Berggren basis [40,49] is generated by a finite-range potential, which can be written as the solutions of the one-body Schröndinger equation in the complex momentum space, which reads

$$
\frac{d^{2} u(k, r)}{d r^{2}}=\left[\frac{l(l+1)}{r^{2}}+\frac{2 m}{\hbar^{2}} U(r)-k^{2}\right] u(k, r),
$$

where $l$ is the orbital angular momentum of the nucleon motion, $m$ is the mass of the nucleon, $r$ stands for the radius, and $\hbar$ is the reduced Planck constant. The momentum $k$ and wave function $u(k, r)$ can be complex. $U(r)$ is the finite-range potential, which is, in practice, a Woods-Saxon (WS) [50] or GHF potential $[33,44]$. When considering protons, the Coulomb potential must be included in $U(r)$. Bound, resonance, and scattering states can then be generated. The eigenenergy of single-particle states in the above equation is complex in general, and reads $\tilde{e}_{n}=k^{2} / 2 m=e_{n}-i \gamma_{n} / 2$, where $n$ denotes the state [40,49]. $e_{n}$ stands for the energy, whereas $\gamma_{n}$ represents the particle decay width, so that $\gamma_{n}=0$ for bound states and $\gamma_{n}>0$ for resonance states. A schematic Berggren basis set of states in the complex $k$-plane is illustrated in Figure 1. The wave function of a resonance state 
is not square-integrable, as its exponential increase in modulus implies that the wave function of a resonance state cannot be normalized with conventional techniques [40,49]. Consequently, one has to rely on the complex scaling method, which has been seen to properly account for the normalization of resonance states [51].

The completeness relation borne by Berggren basis states $[40,49]$ reads

$$
\sum_{n}|n\rangle\left\langle n\left|+\int_{L_{+}}\right| k\right\rangle\langle k| d k=\mathbf{1},
$$

where $|n\rangle$ states are bound states and resonance states inside the $L_{+}$contour of Figure 1. These states are called pole states, as they are the $S$-matrix poles of the finite-range potential. $|k\rangle$ states are scattering states and follow the $L_{+}$contours in the complex $k$-plane, starting from $k=0$ and going to $k \rightarrow+\infty$, as shown in Figure 1. Scattering states initially form a continuum. Hence, in order to be used in numerical applications, the scattering states along the $L_{+}$contour must be discretized with a Gauss-Legendre quadrature $[5,49]$. It has been checked that $10-45$ states per contour are necessary to have converged results $[5,38]$. Once discretized, the Berggren basis is, in effect, the same as that of the harmonic-oscillator (HO) states within the standard SM [5,49]. Concerning resonance states, only narrow resonance states contribute to the physical states, and thus are included in the real calculations, whereas broad resonance states are not included, as they lie below the $L_{+}$contour.

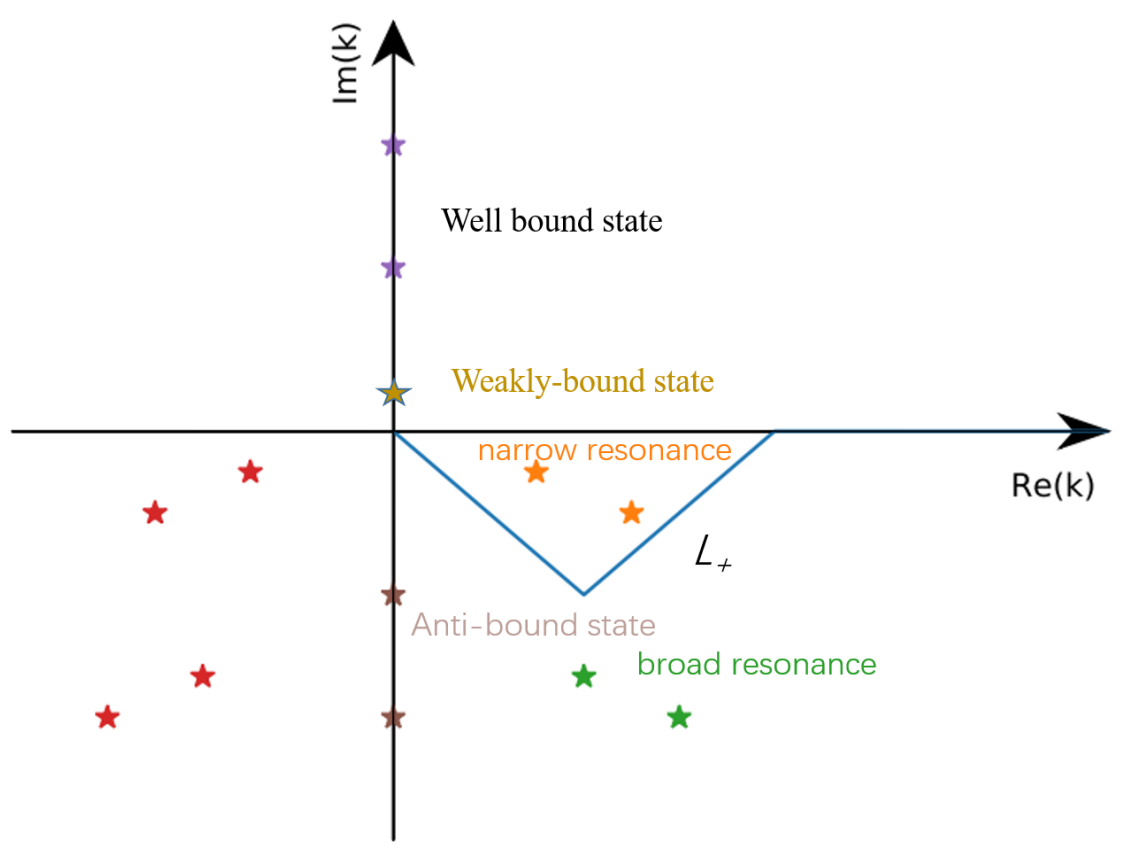

Figure 1. Depiction of the Berggren basis in the complex-momentum- $k$ plane for a fixed partial wave. Typical complex momenta of bound, narrow, and broad-resonance states, i.e., $S$-matrix pole, are provided. The $L_{+}$contour of scattering states encompasses the $S$-matrix poles of interest.

In fact, the Berggren basis is the complex extension of the real-energy completeness relation of Newton [52], which consists of bound states and of a continuum of real-energy scattering states. Contrary to the Newton completeness relation [52], with which, only localized states can be expanded, the Berggren basis can expand unbound resonance states $[40,49]$. The many-body completeness relation is obtained by constructing Slater determinants from the one-body Berggren basis, which contains bound, resonance, and scattering states [5,49]. In the GSM, the Hamiltonian is represented by a complex symmetric matrix when using the one-body Berggren basis, which has to be diagonalized [5,49]. This process can be handled efficiently by using the complex symmetric extension of the Jacobi-Davidson method $[49,53]$, where one can take advantage of the relatively small 
coupling to continuum states in order to have a fast convergence of calculations [5]. The full configuration space is extremely large due to the many scattering states within the model space. In practical calculations, however, we often truncate basis model spaces so that only two particles can occupy scattering states. It has been checked that this is sufficient to obtain converged results for both the energy and decay width of many-body states $[5,38,54]$.

In GSM calculations, an effective Hamiltonian must be constructed. There are two main methods to build the effective Hamiltonian in GSM calculations. One is to construct an effective Hamiltonian based on realistic nuclear force $[38,39,41]$, and hence in the frame of the realistic GSM, whereas the other one consists of using an effective phenomenological nuclear potential $[5,36,37,49]$, in which, the parameters of the potential are optimized to reproduce experimental data. In the following, we give details about these two versions of GSM.

\subsection{Realistic Gamow Shell Model Calculations}

Within realistic GSM, one starts from the intrinsic Hamiltonian of an $A$-body system, which reads

$$
H=\sum_{i=1}^{A} \frac{\boldsymbol{p}_{i}^{2}}{2 m}+\sum_{i<j}^{A} V_{\mathrm{NN}}^{i j}-\frac{\boldsymbol{P}^{2}}{2 A m}
$$

where $\boldsymbol{p}_{i}$ is the nucleon momentum in laboratory frame, $\boldsymbol{P}=\sum_{i=1}^{A} \boldsymbol{p}_{i}$ is the center-ofmass $(\mathrm{CoM})$ momentum of the system, and $V_{\mathrm{NN}}^{(i j)}$ is the realistic NN interaction, such as CD-Bonn [55] and $\mathrm{N}^{3} \mathrm{LO}$ [56] interaction. In the above Hamiltonian, the CoM energy is removed. In order to construct the effective Hamiltonian to be used in GSM calculations, an auxiliary potential is usually introduced $[38,57,58]$, so that the Hamiltonian can be rewritten as,

$$
\begin{aligned}
H \quad & =\sum_{i=1}^{A}\left(\frac{\boldsymbol{p}_{i}^{2}}{2 m}+U\right)+\sum_{i<j}^{A}\left(V_{\mathrm{NN}}^{(i j)}-U-\frac{\boldsymbol{p}_{i}^{2}}{2 A m}-\frac{\boldsymbol{p}_{i} \cdot \boldsymbol{p}_{j}}{A m}\right) \\
& =H_{0}+H_{1}
\end{aligned}
$$

where $H_{0}=\sum_{i=1}^{A}\left(\frac{p_{i}^{2}}{2 m}+U\right)$ has a one-body form, and $H_{1}=\sum_{i<j}^{A}\left(V_{\mathrm{NN}}^{(i j)}-U-\frac{\boldsymbol{p}_{i}^{2}}{2 A m}-\frac{\boldsymbol{p}_{i} \cdot \boldsymbol{p}_{j}}{A m}\right)$ is the residual two-body interaction, including the correction issued from the CoM motion. For the auxiliary potential $U$, we usually take a WS finite-range potential. To speed up the convergence of many-body calculations, the bare force is often softened by a similarity renormalization group method [59], such as the similarity renormalization group (SRG) and $V_{\text {low- } k}$,in order to remove the strong short-range repulsive core.

The realistic NN interaction is firstly defined in a relative momentum space. However, the many-body problem is usually solved in the laboratory frame (with, e.g., the HO basis), so that a transformation from relative and CoM coordinates to the laboratory frame is necessary. This procedure can be conveniently carried out in the HO basis via BrodyMoshinsky brackets [60]. In the $\mathrm{HO}$ basis, the two-body completeness relation reads

$$
\sum_{\alpha \leq \beta}|\alpha \beta\rangle\langle\alpha \beta|=\mathbf{1}
$$

where $|\alpha \beta\rangle$ is the two-particle state of the HO basis. The two-body interaction in the HO basis is given by

$$
V_{\mathrm{HO}}=\sum_{\alpha \leq \beta}^{N_{\text {shell }}} \sum_{\gamma \leq \delta}^{N_{\text {shell }}}|\alpha \beta\rangle\left\langle\alpha \beta\left|V_{\text {low }-k}\right| \gamma \delta\right\rangle\langle\gamma \delta|,
$$

where $N_{\text {shell }}=2 n+l$, indicates that a finite summation is performed up to $N_{\text {shell }}$. The GSM calculations are carried out in the Berggren basis, so that the transformation of the interaction matrix elements from the $\mathrm{HO}$ basis to the Berggren basis needs to be carried 
out. This is achieved, in practice, by computing the overlaps between the Berggren and $\mathrm{HO}$ basis wave functions,

$$
\langle a b|V| c d\rangle \approx \sum_{\alpha \leq \beta}^{N_{\text {shell }}} \sum_{\gamma \leq \delta}^{N_{\text {shell }}}\langle a b \mid \alpha \beta\rangle\left\langle\alpha \beta\left|V_{\text {low }-k}\right| \gamma \delta\right\rangle\langle\gamma \delta \mid c d\rangle,
$$

where $|a b\rangle(|c d\rangle)$ is a two-particle state of the Berggren basis. For identical particles (protonproton or neutron-neutron), the overlap of the two-body state reads

$$
\langle a b \mid \alpha \beta\rangle=\frac{\langle a \mid \alpha\rangle\langle b \mid \beta\rangle-(-1)^{J-j_{\alpha}-j_{\beta}}\langle a \mid \beta\rangle\langle b \mid \alpha\rangle}{\sqrt{\left(1+\delta_{a b}\right)\left(1+\delta_{\alpha \beta}\right)}},
$$

where $J$ is the total angular momentum of the two-particle state, and $j$ is the angular momentum of a single-particle basis state. The $\langle a \mid \alpha\rangle(\langle b \mid \beta\rangle)$ is the overlap of the one-body basis state, and $\delta_{\alpha \beta}$ is the Kronecker delta. For the proton-neutron coupling, the overlap of the two-body state is simply given by

$$
\langle a b \mid \alpha \beta\rangle=\langle a \mid \alpha\rangle\langle b \mid \beta\rangle .
$$

The overlaps of one-body basis states are directly obtained from an integration in $r$-space

$$
\langle a \mid \alpha\rangle=\int d r r^{2} u_{a}(r) R_{\alpha}(r) \delta_{l_{a} l_{\alpha}} \delta_{j_{a} j_{\alpha}} \delta_{t_{a} t_{\alpha}},
$$

where $u(r)$ and $R(r)$ are the radial parts of the single-particle Berggren and HO basis wave functions, with $l, j$, and $t$ being the orbital, total angular momentum, and isospin quantum number, respectively. The single-particle wave functions of resonance and continuum states are not localized and hence are not square-integrable. The transformation defined by Equation (7), in fact, utilizes the short-range nature of nuclear force. Indeed, the Gaussian fall-off of the $\mathrm{HO}$ wave function renders the overlaps integrable, even when one considers resonances or scattering states of complex energy. For long-range operators, such as the one-body kinetic energy and Coulomb potential, using Equation (7) is not suitable in practice. In this case, we use the exterior complex scaling technique [51] to treat the kinetic and Coulomb operator, i.e., terms proportional to $p^{2}$ and $1 / r$, respectively, with the Berggren basis.

The obtained interaction matrix elements in the Berggren basis are complex, and associated operators are non-Hermitian. The many-body perturbation theory (MBPT), named the full $\hat{Q}$-box folded-diagram method [61], is employed to construct the realistic complex effective Hamiltonian in the defined model space for GSM calculations. The complex- $k$ Berggren basis states in the model space are non-degenerate; therefore a nondegenerate $\hat{Q}$-box folded-diagram perturbation, i.e., the extended Kuo-Krenciglowa (EKK) method [62], has been used. For this, we first calculate the $\hat{Q}$-box using MBPT in the Berggren complex- $k$ basis,

$$
\begin{aligned}
\widehat{Q}(E) & =P V P+P V Q \frac{Q}{E-Q H Q} Q V P \\
& =P V P+P V Q \frac{Q}{E-Q H_{0} Q} Q V P+\ldots,
\end{aligned}
$$

where $E$ is starting energy, $P$ and $Q$ represent the model space and the excluded space, respectively, with $P+Q=1$. The $\hat{Q}$-box is composed of irreducible valence-linked diagrams $[57,58]$, which can be built order-by-order. $V$ and $H$ are the two-body interaction and two-body Hamiltonian, respectively, and $H_{0}$ is the unperturbed one-body Hamiltonian. The derivatives of the $\hat{Q}$-box are defined as 


$$
\begin{aligned}
\widehat{Q}_{s}(E) & =\frac{1}{s !} \frac{d^{s} \widehat{Q}(E)}{d E^{s}} \\
& =(-1)^{s} P V Q \frac{Q}{(E-Q H Q)^{s+1}} Q V P,
\end{aligned}
$$

where $s$ denotes the $s$-th derivative.

The effective Hamiltonian $H_{\text {eff }}$ can then be constructed in operator form [63], written as

$$
\widetilde{H}_{\text {eff }}=\widetilde{H}_{B H}(E)+\sum_{k=1}^{\infty} \widehat{Q}_{k}(E) \widetilde{H}_{\text {eff }}
$$

where $\widetilde{H}_{\text {eff }}$ stands for $\widetilde{H}_{\text {eff }}=H_{\text {eff }}-E$, and $\widetilde{H}_{B H}(E)=H_{B H}(E)-E$ is the Block-Horowitz Hamiltonian shifted by an energy $E$, with

$$
\begin{aligned}
H_{B H}(E) & =P H_{0} P+\widehat{Q}(E) \\
& =P H_{0} P+P V P+P V Q \frac{Q}{E-Q H Q} Q V P .
\end{aligned}
$$

$\widetilde{H}_{\text {eff }}$ is obtained by performing iterations of Equation (13), which is equivalent to calculate folded-diagrams, where one considers high-order contributions by summing up the subsets of diagrams to finite order. When convergence is obtained, the effective Hamiltonian is given by $H_{\text {eff }}=\widetilde{H}_{\text {eff }}-E$, and the effective interaction reads $V_{\text {eff }}=H_{\text {eff }}-$ $\mathrm{PH}_{0} P$. The extended $\hat{Q}$-box folded-diagram calculations provide a useful approach for including effects from the continuum coupling and core polarization $[57,58,61,62]$.

\subsection{Gamow Shell Model with Phenomenological Nuclear Potential}

Within the Gamow shell model with phenomenological nuclear potential, the nucleus is assumed to be a system of $N_{v}$ valence particles outside a frozen closed core, from which, core polarization is absent $[5,36,37,49]$. The GSM Hamiltonian, expressed with intrinsic nucleon-core cluster-orbital shell model coordinates [64], writes

$$
H=\sum_{i=1}^{N_{v}}\left[\frac{\boldsymbol{p}_{i}^{2}}{2 \mu_{i}}+U_{\text {core }}(i)\right]+\sum_{i<j=1}^{N_{v}}\left[V(i, j)+\frac{p_{i} p_{j}}{M_{\text {core }}}\right],
$$

where $\boldsymbol{p}_{i}$ is the nucleon momentum in cluster-orbital shell model frame, $U_{\text {core }}$ is the singleparticle nucleon-core potential, and $V$ is the phenomenological $\mathrm{NN}$ interaction between valence nucleons. $\mu_{i}$ and $M_{\text {core }}$ stand for the reduced mass of the nucleon and the mass of the core, respectively. The $\frac{p_{i} p_{j}}{M_{\text {core }}}$ term accounts for the two-body recoil term. As seen in Equation (15), the GSM has two components: the one-body part Hamiltonian $H_{0}=$ $\sum_{i=1}^{N_{v}}\left[\frac{p_{i}^{2}}{2 \mu_{i}}+U_{\text {core }}(i)\right]$ and the two-body part Hamiltonian $H_{I}=\sum_{i<j=1}^{N_{v}}\left[V(i, j)+\frac{p_{i} p_{j}}{M_{\text {core }}}\right]$. The core-valence particle potential $U_{\text {core }}$ is usually a WS potential, in which a spin-orbit term is included. The NN interaction $V$ takes the form of an effective phenomenological NN interaction, such as Minnesota [65], Furutani-Horiuchi-Tamagaki (FHT) [66,67], or effective field theory (EFT) $[18,56]$ interactions. The parameters within the effective Hamiltonian in Equation (15), both one- and two-body interactions, need to be optimized to reproduce experimental data. For optimizations, the $\chi^{2}$ optimization method is employed, where one uses the Gauss-Newton algorithm augmented by the singular value decomposition technique to calculate the Jacobian pseudo-inverse [43]. 


\section{Gamow Shell Model Calculations}

\subsection{Neutron-Rich Oxygen and Fluorine Isotopes}

Neutron-rich oxygen isotopes form a particularly interesting chain for experimental and theoretical research. Firstly, the proton number $Z=8$ shows magical properties for the neutron-rich oxygen isotopes, which provide a good laboratory to perform configuration interaction (shell-model) calculations $[22,38,68,69]$. Secondly, the nuclei ${ }^{22} \mathrm{O}$ and ${ }^{24} \mathrm{O}$ exhibit doubly magicity at the neutron number $N=14$ and 16, respectively, [70-73]. Thirdly, experiments have shown that the ${ }^{25} \mathrm{O}$ and ${ }^{26} \mathrm{O}$ are unbound and decay by one- and twoneutron emission, respectively, [8,74]. Experimental studies suggest that ${ }^{24} \mathrm{O}$ is the heaviest bound isotope of the oxygen chain $[8,74]$. However, the loosely unbound property of ${ }^{26} \mathrm{O}$, which is only $-18 \mathrm{keV}$ unbound [8], is a strong incentive to investigate the bound or unbound character of ${ }^{28} \mathrm{O}$, which should have a magicity of $N=20$. Consequently, the neutron-rich oxygen isotopes provide an ideal laboratory to study many-body correlation, continuum coupling, and single-particle structure. By adding one valence proton to the neutron-rich oxygen isotopes, one obtains the fluorine isotopes at the neutron drip line, which can sustain six additional neutrons after ${ }^{25} \mathrm{~F}$, hence, up to ${ }^{31} \mathrm{~F}$, which is suspected to be at the neutron drip line of the fluorine chain [75]. This dramatic change is called an "oxygen anomaly". Moreover, many exotic properties develop at the neutron drip line for fluorine isotopes, such as halos in ${ }^{29} \mathrm{~F}$ [76] and ${ }^{28} \mathrm{~F}$ within the island of inversion around $N=20$ [77], and thus fluorine isotopes provide a very interesting ground for theoretical studies.

\subsubsection{Realistic Gamow Shell Model Calculations}

We have developed realistic GSM with the Berggren basis using a WS potential, while the realistic effective Hamiltonian is constructed within the model space using a nondegenerate $\hat{Q}$-box folded-diagram method [38]. We first employed it to investigate the neutron-rich oxygen isotopes up to and beyond the neutron drip line [38]. In our calculations, the realistic CD-Bonn potential [55] was used. To speed up the convergence of many-body calculations, the bare force is usually softened to remove the strong short-range repulsive core. The $V_{\mathrm{low}-k}$ method [59] is used for that matter in Ref. [38].

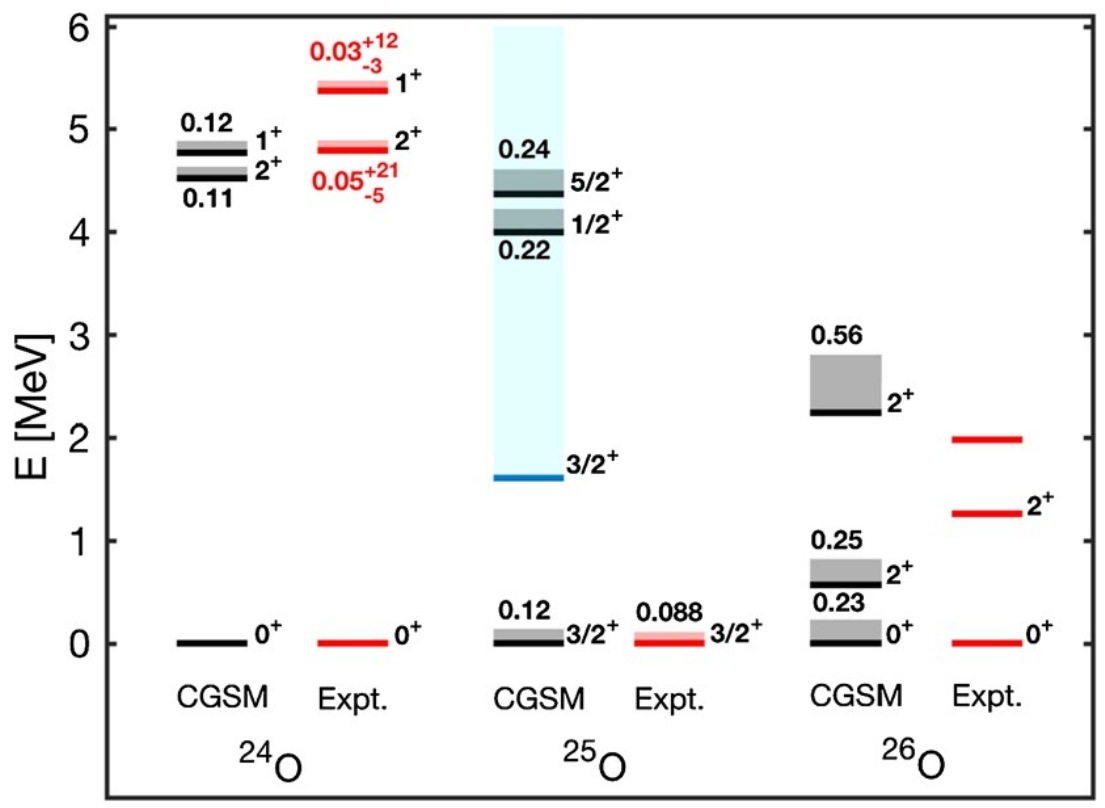

Figure 2. Calculated spectrum of ${ }^{24,25,26} \mathrm{O}$, compared with available experimental data [8,10,74]. The resonances are indicated by shades, and their widths (in $\mathrm{MeV}$ ) are given by the number below or above the levels. The light blue shade indicates the $3 / 2^{+}$many-body scattering states (with permissions from Ref. [38]). The "CGSM" stays for "core Gamow shell model". 
Figure 2 shows the calculated low-lying states of ${ }^{24-26} \mathrm{O}$, along with experimental data $[8,10,74]$. Our realistic GSM calculations [38] reproduce the experimental excitedstate spectrum well, including the observed resonance widths. The ground state energies and one-neutron separation energies $S_{n}$ of the neutron-rich oxygen isotopes are also calculated [38] (see Figure 3) and compared to the experimental data $[8,10,74,78]$. The WS parameters used, taken from Ref. [38], reproduce the experimental $1 s_{1 / 2}$ and $0 d_{3 / 2}$ single-particle energies well, including the decay width of the $0 d_{3 / 2}$ state, but give the $0 d_{5 / 2}$ energy as lower than the experimental data, at about $1.17 \mathrm{MeV}$ [10]. The results presented in Figure 3 show that adopting the experimental $0 d_{5 / 2}$ energy can dramatically improve calculations. Overbinding in the GSM calculations of oxygen isotopes after ${ }^{24} \mathrm{O}$ is obtained in Ref. [38], which is caused by the absence of the three-nucleon force (3NF).
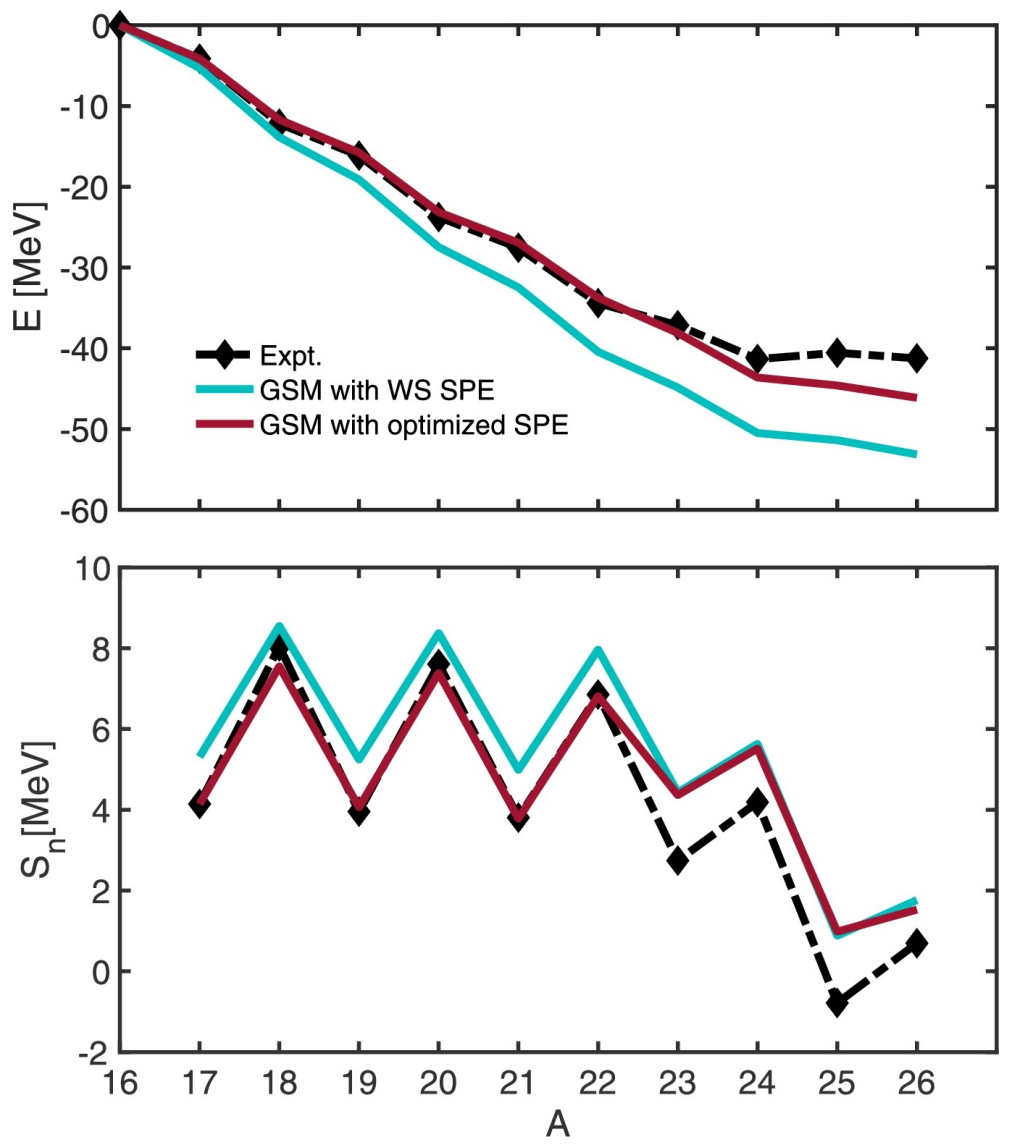

Figure 3. Calculated ground state energies of oxygen isotopes with respect to the ${ }^{16} \mathrm{O}$ core (upper panel) and associated neutron separation energies $S_{n}$ (lower panel) as a function of atomic number compared with experimental data $[8,74,78]$. "GSM with WS SPE" indicates that the calculations were performed with Woods-Saxon (WS) single-particle energies (SPE), whereas "GSM with optimized SPE" means that the calculations were performed with the $0 d_{5 / 2}$ SPE replaced by its experimental value (with permissions from Ref. [38]).

\subsubsection{Ab-initio Realistic GSM Calculations within GHF Basis}

GSM is usually performed using a basis generated by a WS potential [5,19,36-39], whose parameters must be determined by fitting experimental single-particle energies and resonance widths. However, the single-particle energies and resonance widths in the multishell case are sometimes difficult to assess due to the lack of experimental data for that matter [10]. We then developed an ab initio realistic GSM approach by introducing the GHF basis as the Berggren basis [41]. The GHF basis is obtained by using the same interaction as the one used in the construction of the effective SM Hamiltonian [41], and thus there is no parameter introduced in the GHF Berggren basis. Starting from the chiral next-to-next-toleading-order $\left(\mathrm{NNLO}_{\mathrm{opt}}\right.$ ) force [79], we perform a nondegenerate $\hat{Q}$-box folded-diagram 
calculation [38,62] in the GHF basis in order to construct a complex effective Hamiltonian. The energies and widths of single-particle orbitals can also be obtained self-consistently using the nondegenerate $\hat{S}$-box folded-diagram method [41]. The neutron-rich fluorine isotopes have been extended to the $p f$-shell, using a cross-shell effective Hamiltonian with the following model space : $\left\{1 s_{1 / 2}, 0 d_{5 / 2}, 0 d_{3 / 2}\right\}$ for the valence proton, and $\left\{1 s_{1 / 2}, 0 d_{3 / 2}+\right.$ $d_{3 / 2}$ scattering states, $1 p_{3 / 2}+p_{3 / 2}$ scattering states, $1 p_{1 / 2}+p_{1 / 2}$ scattering states, $f_{7 / 2}$ scattering states $\}$ for valence neutrons. More details can be found in Ref. [41]. The constructed effective Hamiltonian was employed to study neutron-rich oxygen and fluorine drip line nuclei.

Figure 4 shows the calculated ground-state energies and neutron separation energies $S_{n}$ of oxygen and fluorine isotopic chains, as well as comparisons with experimental data [78] and other theoretical calculations [31,68,79-83]. The GSM calculations using a GHF basis and based on the $\mathrm{NNLO}_{\text {opt }}$ [79] provide the correct location of the neutron drip line of oxygen isotopes and a good description of the unbound nuclei ${ }^{25,26} \mathrm{O}$, which lie beyond the neutron drip line (see the left panel of Figure 4). Note that, when using the standard SM calculations with the USDB interaction [68], conventional SM calculations with NN + 3NF [82], or valence-space IMSRG (VS-IMSRG) calculations with NN + $3 \mathrm{NF}$ [81], the resonance and continuum couplings are absent. Complex CC [31] and GSM calculations [80] are displayed in Figure 4 for comparison.
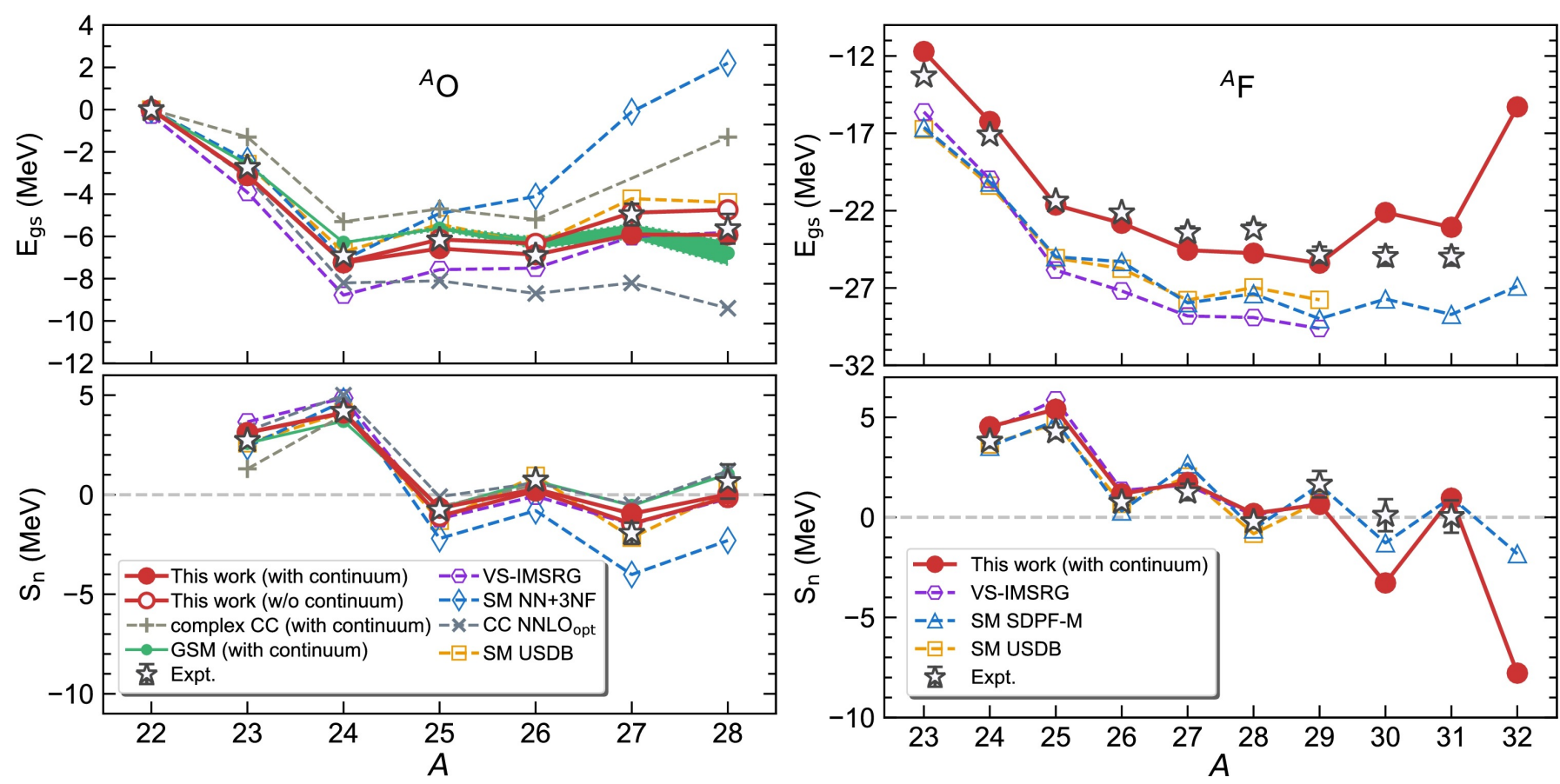

Figure 4. Calculated ground-state energies (upper panel) with respect to the ${ }^{22} \mathrm{O}$ core and associated neutron separation energies $S_{n}$ (lower panel) for oxygen and fluorine isotopes, compared with experimental data [78] (the AME2016 extrapolated values are taken for ${ }^{27,28} \mathrm{O}$ and ${ }^{30,31} \mathrm{~F}$ ) and theoretical calculations from other groups: complex coupled-cluster (CC) with next-to-next-to-next-to-leading-order nucleon-nucleon CC with $\mathrm{NNLO}_{\text {opt }}$ interaction [79], GSM [80], -space in-medium similarity renormalization group (VS-IMSRG) [81], SM with NN+3NF [82], SM with USDB [68], and SM with SDPF-M [83] (with permissions from Ref. [41]).

The results of fluorine isotopes are shown in the right panels of Figure 4. For comparison, standard SM calculations using USDB [68] and SDPF-M [83] effective interactions are also presented. All ground-state energies in Figure 4 are given with respect to the ground state of ${ }^{22} \mathrm{O}$. Experiments revealed that ${ }^{31} \mathrm{~F}$ is a neutron drip line nucleus [75]. Although our GSM calculations provide a lower energy of ${ }^{31} \mathrm{~F}$ compared to that of ${ }^{30} \mathrm{~F},{ }^{31} \mathrm{~F}$ is still unbound compared to ${ }^{29} \mathrm{~F}$. However, our GSM calculations provide good descriptions of ground-state energies for ${ }^{23-29} \mathrm{~F}$. 


\subsubsection{GSM Calculations with Phenomenological Nuclear Potential}

Many ab initio calculations, such as SM [82], VS-IMSRG [81], complex CC [31], and realistic GSM $[38,39,41]$ calculations, have been employed for the description of neutronrich oxygen and fluorine isotopes. However, these calculations bear a large theoretical uncertainty. Furthermore, results arising from ab initio calculations depend on the realistic nuclear forces used (a short summary of the VS-IMSRG calculations based on different chiral nuclear forces can be found in Ref. [80]). Moreover, continuum coupling is absent in the VS-IMSRG [81] and SM [82] calculations. Similar situations also occur for neutron-rich fluorine isotopes, where few calculations have been performed and most of the calculations are absent for the continuum coupling $[83,84]$. Based on these grounds, we performed the GSM calculations with a phenomenological nuclear interaction for neutron-rich oxygen and fluorine drip line nuclei.

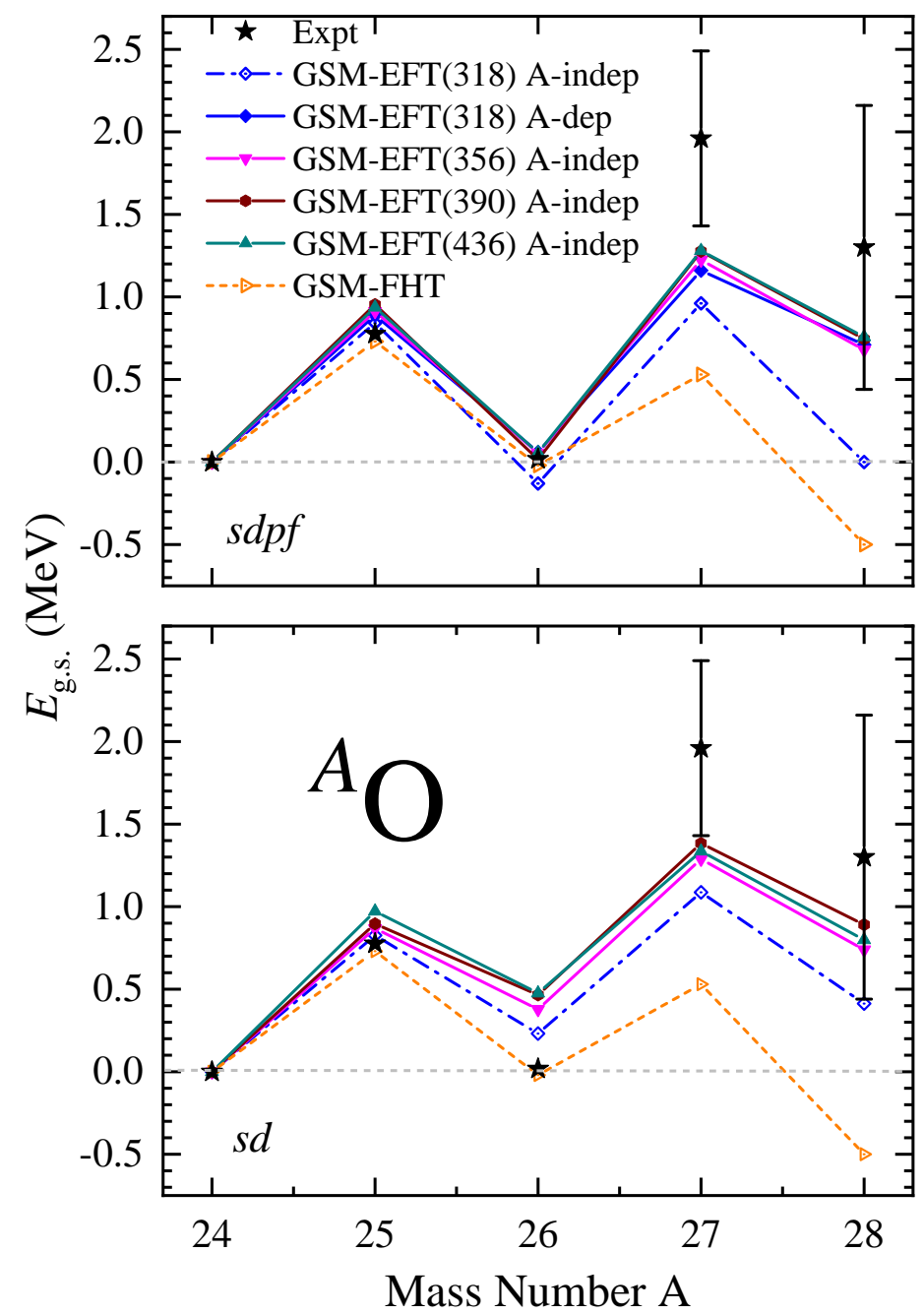

Figure 5. Energies of ground states in ${ }^{24-28} \mathrm{O}$, calculated by GSM within the $\operatorname{sdpf}\left(s_{1 / 2}, p_{1 / 2,3 / 2}\right.$, $d_{3 / 2,5 / 2}, f_{5 / 2,7 / 2}$ partial waves) (upper) and $s d\left(s_{1 / 2}, d_{3 / 2,5 / 2}\right.$ partial waves) (lower) model spaces, using the effective field theory (EFT) EFT(318)(the value within braket stands momentum cutoffs), EFT(356), EFT(390), EFT(436), and Furutani-Horiuchi-Tamagaki (FHT) interactions, with A-independence ( $A$-indep) or A-dependence ( $A$-dep) (see details in Ref. [42]). Results are compared with the experimental data available, represented by a star. The data for ${ }^{25,26} \mathrm{O}$ and ${ }^{27,28} \mathrm{O}$ are taken from experiment (see Refs. [8,74]) and evaluations given in AME2016 [78] (with permissions from Ref. [42]).

For the considered neutron-rich oxygen isotopes, the closed-shell nucleus ${ }^{22} \mathrm{O}$ is selected to be the inner core. The one-body potential is mimicked by a WS potential, whose 
parameters are adjusted to reproduce the single-particle spectrum of ${ }^{23} \mathrm{O}$ [10]. We use the pionless EFT interaction $[85,86]$ as the two-body interaction. Owing to the few available data related to the oxygen drip line nuclei [10], only the leading order (LO) NN interaction of the EFT force is fitted to reproduce selected experimental data. The effect of the $3 \mathrm{NF}$ at LO is then effectively taken into account in the fitted parameters. Details can be found in Ref. [42]. We calculated the energies of the ground states of ${ }^{24-28} \mathrm{O}$ with GSM within $s d p f$ $\left(s_{1 / 2}, p_{1 / 2,3 / 2}, d_{3 / 2,5 / 2}, f_{5 / 2,7 / 2}\right.$ partial waves) and $s d\left(s_{1 / 2}, d_{3 / 2,5 / 2}\right.$ partial waves) active spaces, using different EFT interactions (see details in Ref. [42]). The calculated groundstate energies of ${ }^{24-28} \mathrm{O}$ are shown in Figure 5. The calculations within the $s d$ space show that the ${ }^{25-28} \mathrm{O}$ isotopes are unbound and that their binding energies are close to the experimental data $[8,74,78]$ and to calculations performed within the $\operatorname{ddp} f$ space. However, the calculations obtained in the $s d$ space provide an unbound ${ }^{26} \mathrm{O}$ ground state, by about $300 \mathrm{keV}$ relative to the ground state of ${ }^{24} \mathrm{O}$, which is a little higher than its experimental value, which is about $20 \mathrm{keV}$ unbound [8]. Though the energy difference obtained using the two different model spaces is small, the calculation performed within the spdf space seems to be more reasonable. The GSM calculations performed within the $s d p f$ space provide good agreements of the ${ }^{23-26} \mathrm{O}$ ground states with experimental data $[8,74,78]$; in particular, the two-neutron separation energy $\left(S_{2 n}\right)$ of ${ }^{26} \mathrm{O}$ is about $20 \mathrm{keV}$ [8]. The calculated ground state of ${ }^{28} \mathrm{O}$ is unbound in all three cases and located about $700 \mathrm{keV}$ above the ground state of ${ }^{24} \mathrm{O}$. The ground states of the ${ }^{26,28} \mathrm{O}$ isotopes are unbound, but bear negligible widths. Together with the calculated one-body densities of the ${ }^{26,28} \mathrm{O}$ isotopes in Ref. [42], the results suggest that the ground state of ${ }^{28} \mathrm{O}$ exhibits four-neutron decay by way of $2 n-2 n$ emission via the ${ }^{26} \mathrm{O}$ ground state, which is consistent with few-body [87] and the above ab initio GSM calculations [41].

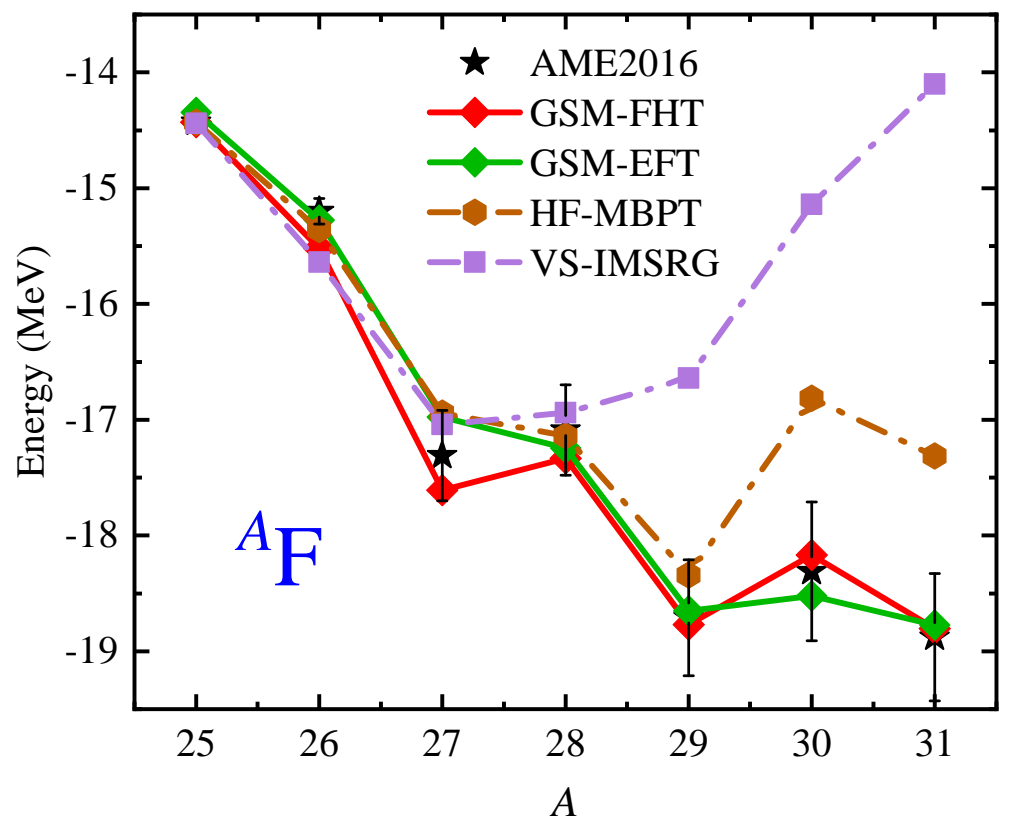

Figure 6. Energies of ${ }^{25-31} \mathrm{~F}$ with respect to the ${ }^{24} \mathrm{O}$ core, calculated within different theoretical frameworks and compared to experimental data [78]. Besides the GSM calculations using FHT and EFT interactions, calculations in the Hartree-Fock many-body perturbation theory (HF-MBPT) [88] and VS-IMSRG [84] frameworks, utilizing the harmonic-oscillator $(\mathrm{HO})$ basis, hence being without continuum coupling, are presented (with permissions from Ref. [18]).

Figure 6 shows the GSM calculations of the binding energies of fluorine isotopes using FHT and EFT interactions (see details in Ref. [18]), wich are compared with experimental data [78] and Hartree-Fock MBPT (HF-MBPT) [88] and VS-IMSRG [84] calculations, which are both performed in the $\mathrm{HO}$ basis. The energy of ${ }^{25} \mathrm{~F}$ has been fixed to its experimental datum in all used models in Figure 6. We can see that all calculations reproduce the 
ground state energies of ${ }^{25-28} \mathrm{~F}$ isotopes well, situated in the well-bound region, whereas differences start after ${ }^{29} \mathrm{~F}$, i.e., when one reaches the neutron drip line. Due to the absence of both multi-shell and continuum couplings, the VS-IMSRG calculations [84] provide visible differences, which are about $4-$ to $5-\mathrm{MeV}$ in magnitude for ${ }^{30,31} \mathrm{~F}$. When applying the HF-MBPT method [88], the cross-shell couplings generated by the $s d$ and $p f$ shells are included, so that proper binding energies of up to ${ }^{29} \mathrm{~F}$ are predicted. However, due to the lack of continuum coupling, the binding energies of ${ }^{30,31} \mathrm{~F}$ are about $1 \mathrm{MeV}$ away from experimental data. The GSM calculation performed with FHT and EFT interactions correctly provides binding energies of up to ${ }^{31} \mathrm{~F}$. Moreover, the odd-even staggering encountered from the ${ }^{28} \mathrm{~F}$ isotope, typical of the presence of a strong proton-neutron interaction, is well reproduced, with ${ }^{30} \mathrm{~F}$ being unbound and ${ }^{31} \mathrm{~F}$ being loosely bound in our calculations.

Recent realistic shell model calculations [89] have pointed out that nuclear deformation plays an important role in the neutron drip line nuclei. Within GSM, deformation can be accounted for by configuration mixing using a cross-shell valence space [5]. Besides deformation, continuum coupling also gives important contributions in drip line nuclei. They are strongly coupled with continuum states near the particle-emission threshold, which provides additional binding energy [5]. This situation is unlike that occurring in well-bound systems, where one only has strong coupling with nearby deeply-bound single-particle states.

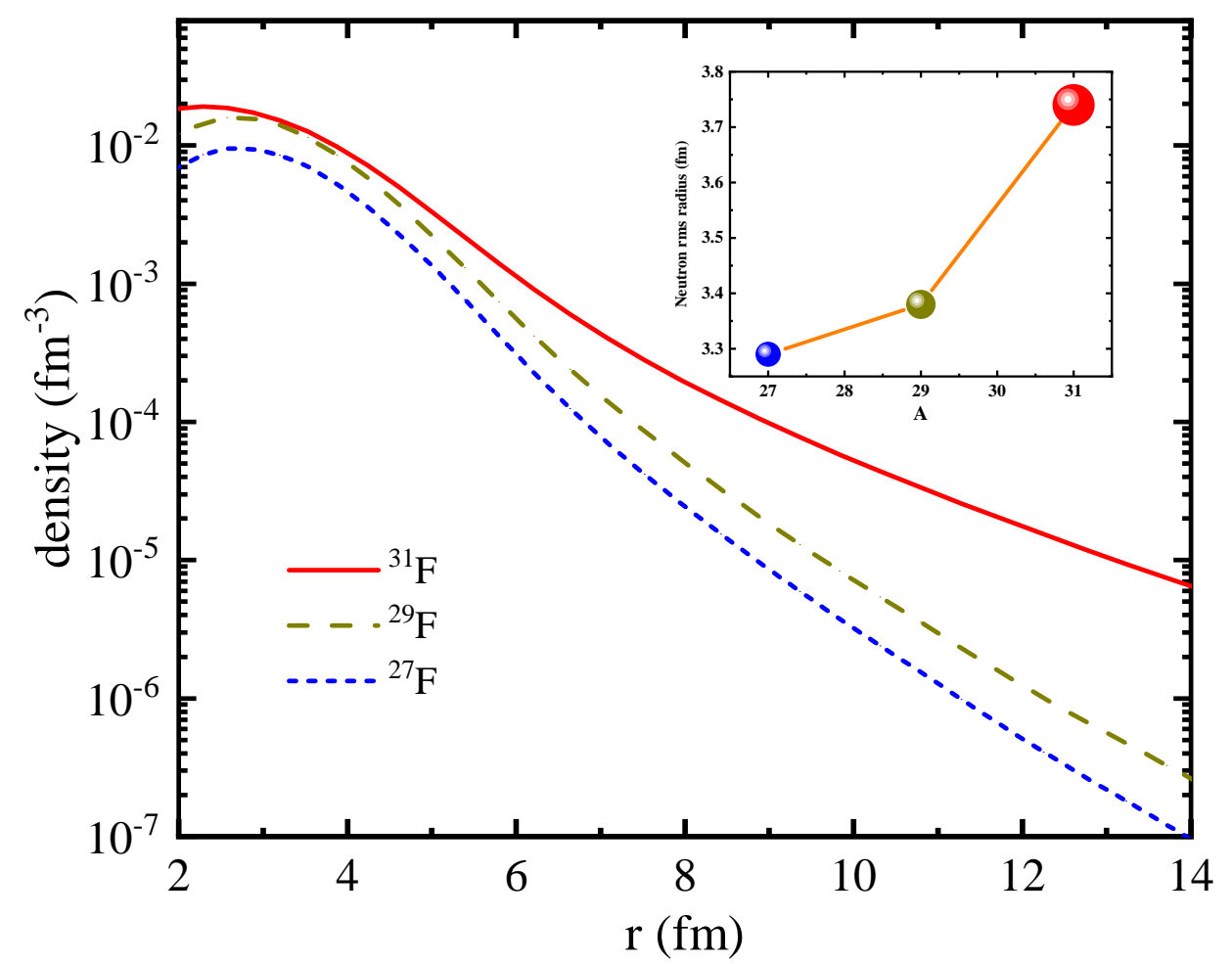

Figure 7. One-nucleon densities of the bound ${ }^{27,29,31} \mathrm{~F}$ isotopes calculated with the GSM using the EFT interaction in the valence space as a function of radii $r$, respectively, depicted by short-dashed, long-dashed, and solid lines. The rms radii of these isotopes are shown in the inset (with permissions from Ref. [18]).

The two-neutron separation energy $S_{2 n}$ of ${ }^{31} \mathrm{~F}$ is about $170 \mathrm{keV}$ [78], which is sufficiently small for sustaining a two-neutron halo. We calculated the one-nucleon densities and neutron rms radii of the neutron-bound ${ }^{27,29,31} \mathrm{~F}$ isotopes using GSM with the EFT interaction (see Figure 7). From our calculations, a halo clearly develops in the asymptotic region of ${ }^{31} \mathrm{~F}$. Indeed, on the one hand, the one-nucleon density of ${ }^{31} \mathrm{~F}$ slowly decreases on the real axis and is about one to two orders of magnitude larger than those of ${ }^{27,29} \mathrm{~F}$ in 
the asymptotic region. Added to that, on the other hand, the neutron rms radius of ${ }^{31} \mathrm{~F}$ does not follow the trend noticed in ${ }^{27,29} \mathrm{~F}$, as the rms radius sharply increases compared to the associated values in ${ }^{27} \mathrm{~F}$ and ${ }^{29} \mathrm{~F}$. Consequently, one can assume from these GSM calculations [18] that ${ }^{31} \mathrm{~F}$ is a two-neutron halo state.

\subsection{Realistic Gamow Shell Model Calculations of Neutron-Rich Calcium Isotopes}

The long chain of calcium isotopes provides an ideal laboratory for both theoretical and experimental investigations of unstable isotopes. With two typical doubly magic isotopes, ${ }^{40} \mathrm{Ca}$ and ${ }^{48} \mathrm{Ca}$, and two new magic isotopes discovered in the neutron-rich region, ${ }^{52} \mathrm{Ca}$ [90] and ${ }^{54} \mathrm{Ca}$ [91], the calcium chain is speculated to end the ${ }^{70} \mathrm{Ca}$ isotope. Its rich nuclear structure data [10] attract continued theoretical interest, especially using methods that include continuum coupling. The realistic GSM based on the realistic CD-Bonn [55] interaction has also been performed to investigate the properties of neutron-rich calcium isotopes up to the drip line.
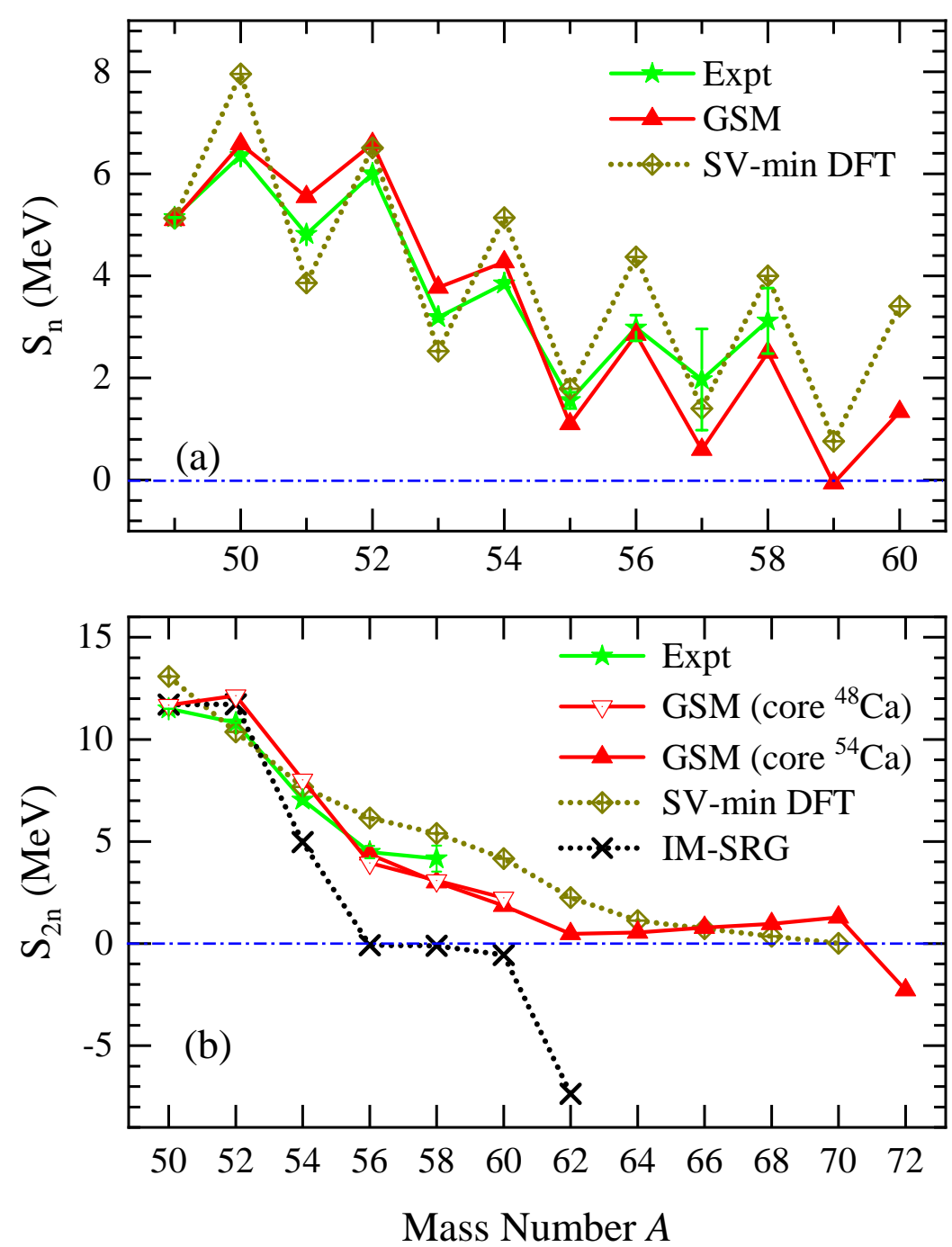

Figure 8. Calculated one-neutron separation energies $S_{n}\left(\right.$ a) and two-neutron separation energies $S_{2 n}$ (b), compared with data [78,92], and calculations obtained with SV-min density-functional theory (DFT) [93] and multireference IM-SRG ( $S_{2 n}$ only) [94]. The $S_{n}$ calculations end at ${ }^{60} \mathrm{Ca}$ because odd isotopes heavier than ${ }^{60} \mathrm{Ca}$ become unbound in our GSM calculations (with permissions from Ref. [47]). 
The calculated one-neutron separation energies $S_{n}$ and two-neutron separation energies $S_{2 n}$ are shown in Figure 8 and compared with experimental data [78,92], DFT [93], and IM-SRG [94] calculations. The calculated one-neutron separation energies $S_{n}$ show that ${ }^{57} \mathrm{Ca}$ is the heaviest odd-mass bound calcium isotope, which is consistent with MBPT calculations [95]. ${ }^{59} \mathrm{Ca}$ is weakly unbound with a small one-neutron separation energy $S_{n}=-326 \mathrm{keV}$ in our GSM calculations. For the two-neutron separation energy $S_{2 n}$, the GSM calculations are performed with two different cores, ${ }^{48} \mathrm{Ca}$ and ${ }^{54} \mathrm{Ca}$. For ${ }^{56,58,60} \mathrm{Ca}$, the two calculations give similar results. The calculated two-neutron separation energy $S_{2 n}$ is in good agreement with experimental data $[78,92]$ and other theoretical calculations, e.g., with DFT [93] and IM-SRG [94] calculations. The large decrease in $S_{2 n}$ at neutron number $N=32$ and 34 indicate that subshell closures occur therein, which has also been suggested from experiments $[90,91]$ and theoretical calculations [94-96]. Moreover, the calculated two-neutron separation energy $S_{2 n}$ using GSM predicts that the two-neutron drip line of the calcium isotopes should be located at ${ }^{70} \mathrm{Ca}$. This is consistent with the recent mean-field calculations of Ref. [97].

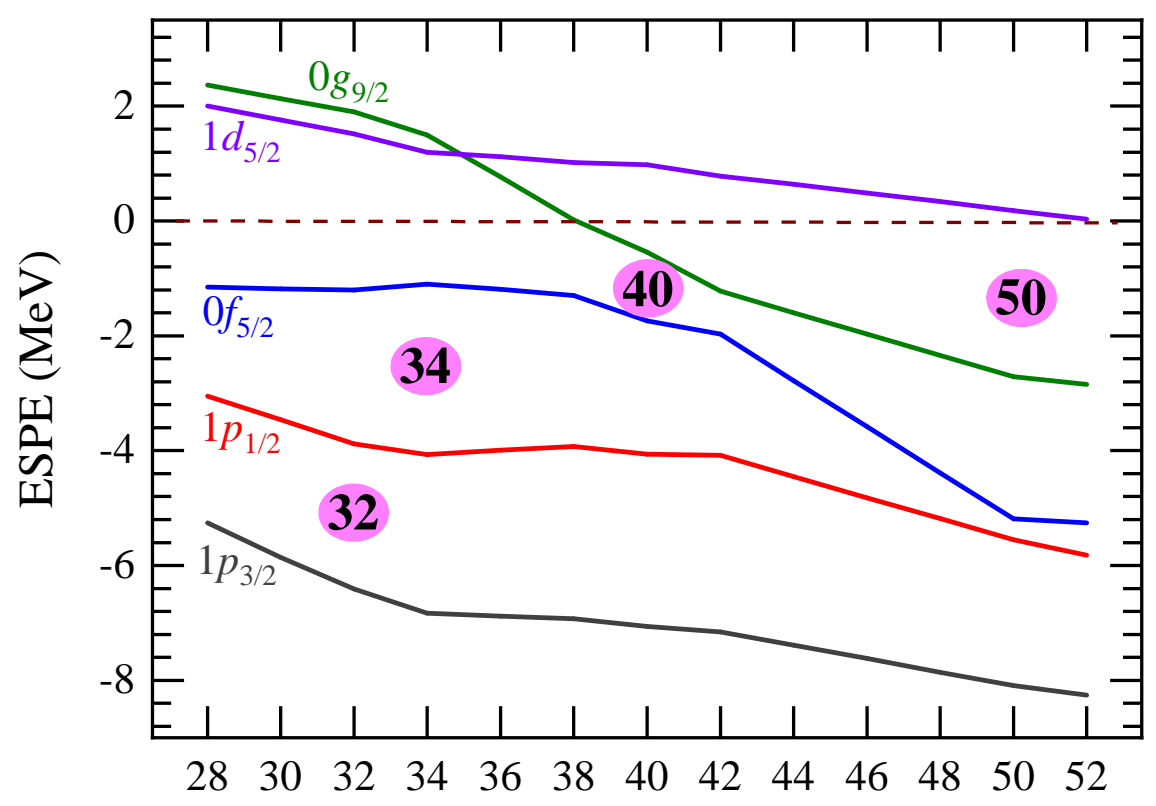

\section{Neutron Number $N$}

Figure 9. Neutron effective single-particle energies (ESPE) with respect to the ${ }^{48} \mathrm{Ca}$ core, as a function of neutron number. The $\mathrm{V}_{\text {low }-k} \Lambda=2.6 \mathrm{fm}^{-1} \mathrm{CD}$-Bonn interaction is utilized (with permissions from Ref. [47]).

In order to see the shell evolution of the calcium isotopes around the neutron number $N=32,34,40$, and 50, we calculated effective single-particle energies (ESPE) based on the GSM effective Hamiltonian. Figure 9 shows the evolution of the valence neutron ESPEs when increasing the neutron number. The calculations show that large shell gaps between $1 p_{3 / 2}$ and $1 p_{1 / 2}$ and between $1 p_{1 / 2}$ and $0 f_{5 / 2}$ exist, indicating that shell closures occur at $N=32$ and 34 , respectively. These results are consistent with experimental observations [90,91] and theoretical calculations [94-96,98]. The shell gap above the $0 f_{5 / 2}$ orbit is reduced at around $N=40$, implying a weakening of the $N=40$ shell closure in the calcium chain. The $0 g_{9 / 2}$ shell becomes bound at $N \geq 40$, which can enhance the stability of the heavy calcium isotopes. The observed ${ }^{60} \mathrm{Ca}$ isotope in experiments [99] may be an indication of this enhanced stability. Moreover, the calculated ESPEs show a significant shell gap at $N=50$, implying a shell closure at ${ }^{70} \mathrm{Ca}$. 


\subsection{One-Proton and Two-Proton Decays in ${ }^{16} \mathrm{Ne}$ and ${ }^{18} \mathrm{Mg}$ Unbound Nuclei}

Two-proton decay is one of the most important drip-line phenomena. It occurs in proton drip line nuclei, such as ${ }^{48} \mathrm{Ni},{ }^{54} \mathrm{~F},{ }^{54} \mathrm{Zn},{ }^{76} \mathrm{~K},{ }^{16} \mathrm{Ne}$, and ${ }^{19} \mathrm{Mg}$ (see a review of this topic in Ref. [4] ). While ${ }^{18} \mathrm{Mg}$ has not been observed, it can decay in principle by proton and/or two-proton emissions. The GSM is then a suitable method to study these types of particle emissions. We carried out GSM calculations of the proton-rich carbon isotones of ${ }^{14} \mathrm{O}$, which are all resonance [10], using ${ }^{14} \mathrm{O}$ as an inner core. The obtained energy spectra of carbon isotones are depicted in Figure 10 with respect to the ground state of ${ }^{14} \mathrm{O}$. One can see that both the energies and widths of experimentally known eigenstates are well reproduced for the low-lying states in ${ }^{15} \mathrm{~F}$ and ${ }^{16} \mathrm{Ne}$ [10]. We also provide predictions for the ${ }^{17} \mathrm{Na}$ and ${ }^{18} \mathrm{Mg}$ nuclear spectra, of which, there are no experimental data. Our calculations show that the ${ }^{16} \mathrm{Ne}$ and ${ }^{18} \mathrm{Mg}$ isotopes are unbound nuclei, where both oneproton separation energies $S_{p}$ and two-proton separation energies $S_{2 p}$ are negative, thereby indicating that two different particle-emission channels are open therein.

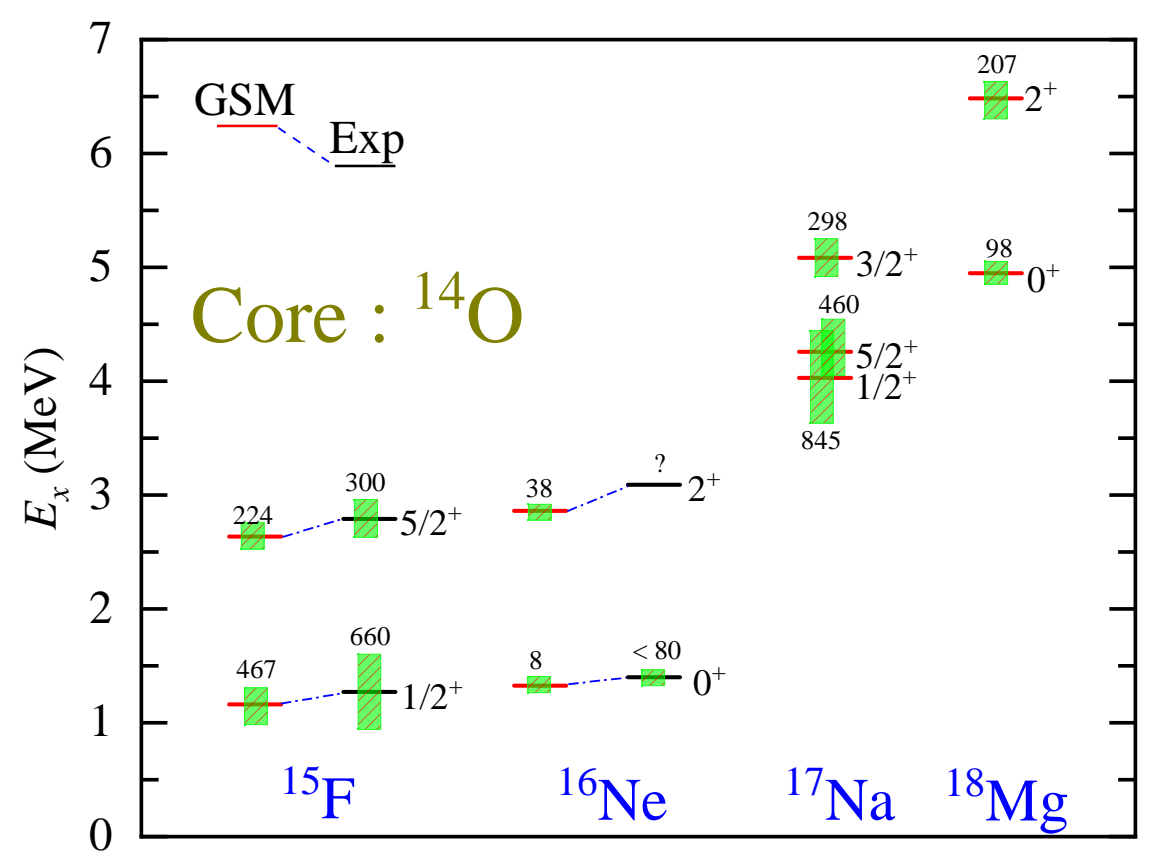

Figure 10. Excitation energies, $E_{x}$, and widths (in $\mathrm{keV}$ ) of the ground and excited states of carbon isotones. The GSM calculations are compared to available experimental data [10,100-102]. Energies are given with respect to the ${ }^{14} \mathrm{O}$ core. Widths are represented by green striped squares, and their explicit values are written above (with permissions from Ref. [48]).

To evaluate one-proton and two-proton decay widths, we changed the central potential depth $V_{0}$ of the WS core potential in order for the $S_{p}$ to become positive or very negative (see details in Ref. [48]). Consequently, it is possible to find a central potential depth for which only the two-proton decay channel is open, so that the obtained width is that of the two-proton emission. The obtained results are shown in Figure $11 . \mathrm{As}^{15} \mathrm{~F}$ and ${ }^{17} \mathrm{Ne}$ are one-proton resonances, their width increases steadily with the Hamiltonian central potential depth. In contrast, one can see that the widths of ${ }^{16} \mathrm{Ne}$ and ${ }^{18} \mathrm{Mg}$ increase abruptly when the one-proton channel opens. The width of two-proton decay is almost constant with respect to the central potential depth below the one-proton emission threshold, and is also about $500 \mathrm{keV}$ to $1 \mathrm{MeV}$ above (see Figure 11). It is reasonable to assume that the two-proton decay width is almost independent of energy. Therefore, the GSM results shown in Figure 11, where only the two-proton channel is open, can be extrapolated to the physical case (indicated by an arrow in Figure 11). This two-proton decay width is about $10-15 \mathrm{keV}$ for both ${ }^{16} \mathrm{Ne}$ and ${ }^{18} \mathrm{Mg}$ nuclei. The one-proton width can be assumed as 
the difference between the total width and the two-proton emission width of 10-15 keV. Then, our calculations show that one-proton emission is negligible for ${ }^{16} \mathrm{Ne}$, whereas the one-proton decay width in ${ }^{18} \mathrm{Mg}$ is estimated to be about $85-90 \mathrm{keV}$. The obtained data for ${ }^{16} \mathrm{Ne}$ are also in agreement with experimental data [10,100-102].
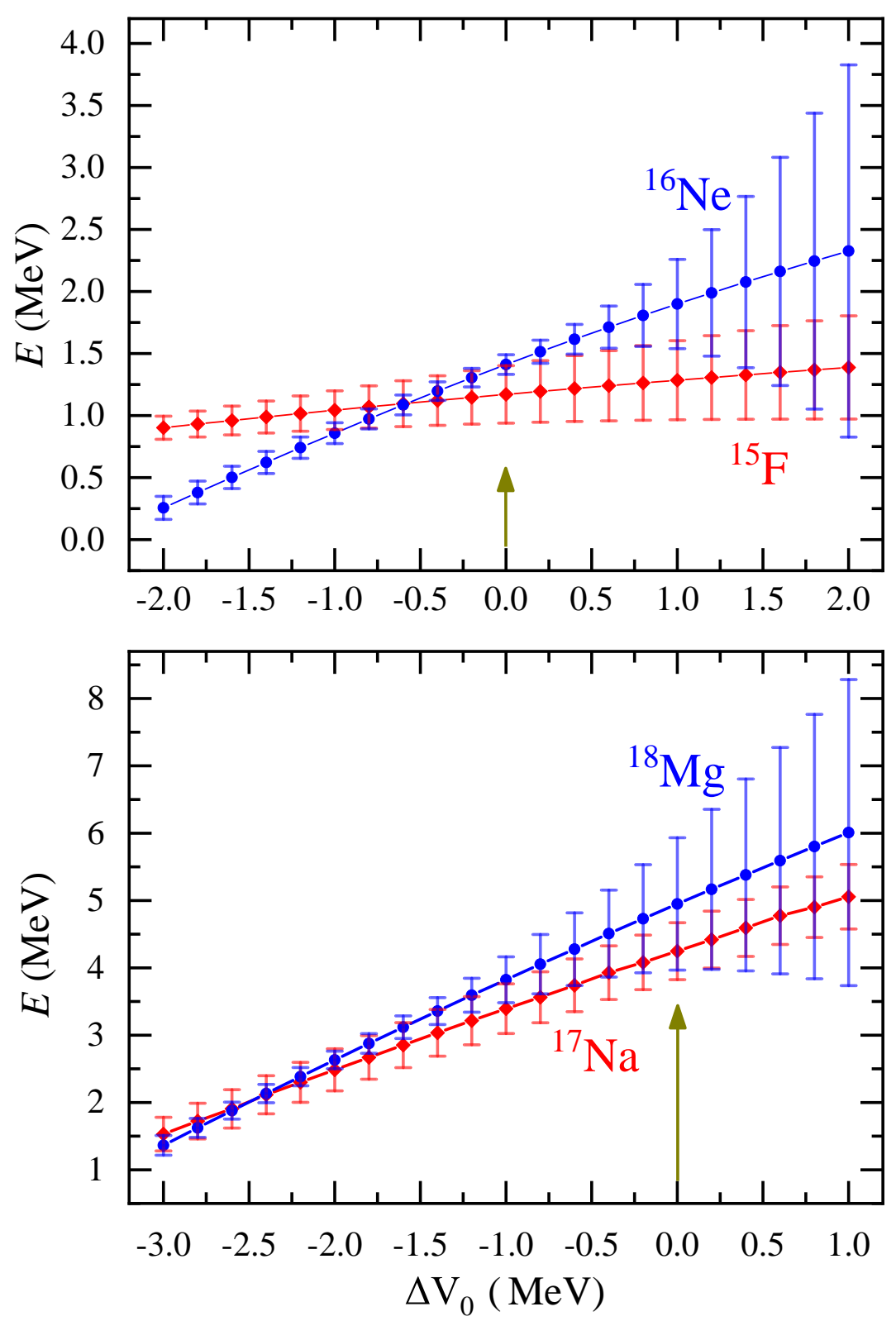

Figure 11. Calculated energies and widths (in $\mathrm{MeV}$ ) of ${ }^{15} \mathrm{~F},{ }^{16} \mathrm{Ne}$ (upper panel), ${ }^{17} \mathrm{Na}$, and ${ }^{18} \mathrm{Mg}$ (lower panel) as a function of the difference $\Delta V_{0}=V_{0}-V_{0}^{\text {(fit) }}$ (fit) of the WS central potential depths (see details in Ref. [48]). Energies are depicted by blue disks and red lozenges for even and odd nuclei, respectively. Widths are represented by segments centered on disks and lozenges. The widths of ${ }^{16} \mathrm{Ne}$ and ${ }^{18} \mathrm{Mg}$ have been multiplied by 20 for readability. Energies are given with respect to the ${ }^{14} \mathrm{O}$ core. The physical GSM calculation, for which $V_{0}=V_{0}^{(\mathrm{fit})}$, is indicated by an arrow (with permissions from Ref. [48]).

\section{Summary}

The Gamow shell model (GSM) is a powerful method for the description of the weakly bound and resonance properties of drip line nuclei. In the present review, we presented several recent applications of GSM dedicated to the study of drip line nuclei, including 
GSM calculations of neutron-rich oxygen and fluorine drip line nuclei, of the long chain of neutron-rich calcium isotopes, and of the unbound proton-rich ${ }^{16} \mathrm{Ne}$ and ${ }^{18} \mathrm{Mg}$ isotopes. For the neutron-rich oxygen and fluorine drip line nuclei, both the realistic GSM and GSM with phenomenological forces have been utilized. Our calculations have described the weakly-bound and unbound properties of drip line nuclei well. Furthermore, the unbound properties of the ${ }^{28} \mathrm{O}$ are obtained within the two both types of GSM calculations, and the two-neutron halo property of ${ }^{31} \mathrm{~F}$ has been predicted in GSM calculations as well. The realistic GSM calculations provide good agreements of the neutron-rich calcium isotopes with experimental data, as GSM calculations predict that the one- and two-neutron drip line nuclei of calcium isotopes are ${ }^{57} \mathrm{Ca}$ and ${ }^{70} \mathrm{Ca}$, respectively. For the unbound proton-rich ${ }^{16} \mathrm{Ne}$ and ${ }^{18} \mathrm{Mg}$ nuclei, GSM calculations provide calculations and predictions for their lowlying spectra. Added to that, the one- and two-proton emission widths could be estimated for ${ }^{16} \mathrm{Ne}$ and ${ }^{18} \mathrm{Mg}$ isotopes. Our calculations have shown that ${ }^{16} \mathrm{Ne}$ decays only by twoproton emission, whereas ${ }^{18} \mathrm{Mg}$ can decay through both one- and two-proton emission channels, whose widths are estimated to be about $85-90$ and $10-15 \mathrm{keV}$, respectively.

GSM has thus been shown to be the tool of choice for the study of drip line nuclei. Many challenges remain to be overcome for the future applications of GSM:

- Due to the large computational cost of the GSM calculations, the GSM has been applied for the neutron-rich nuclei with only one or two valence protons, and, for proton-rich nuclei, with only one or two valence neutrons in the non-resonant continuum. For example, the model space dimension of ${ }^{31} \mathrm{~F}$ is about $10^{7}$ with two valence particles in the continuum. It can easily reach $10^{10}$ without truncations, which is untractable numerically. In the nuclear chart, most of the drip line nuclei need to be described with many valence particles (protons and neutrons). One can think of the neutron-rich $\mathrm{Ne}$ and $\mathrm{Mg}$ drip line isotopes, where both continuum coupling and strong internucleon correlations must be treated properly. These isotopes will provide a challenge for future GSM calculations, due to the large dimensions;

- The diagonalization of the GSM Hamiltonian in order to obtain eigenstates of large resonance widths, such as the second $0_{2}^{+}$state in ${ }^{8} \mathrm{He}$, is very difficult from a numerical point of view;

- The dimensions of the GSM Hamiltonian matrices increase extremely quickly when one adds valence particles, and thus the treatment of the many-body Hamiltonian is difficult when using the configuration interaction framework. Other kinds of manybody methods are urgently needed. The two-particle reduced density matrix method is one of the promising methods to solve the dimensionality problem of the GSM many-body Hamiltonian [103];

- The unbound single-particle states of $s$ waves in neutron-rich nuclei are anti-bound states, which are difficult to include in many-body GSM calculations. The consideration of many-body anti-bound states in GSM (the ground state of ${ }^{10} \mathrm{Li}$ is supposed to be anti-bound, for example [104]) is thus also a challenge for future applications of GSM.

Author Contributions: Writing—original draft preparation, J.L.; writing—review and editing, F.X. and N.M.; critically review, Y.M., B.H., Z.S. and W.Z. All authors have read and agreed to the published version of the manuscript.

Funding: This work has been funded by the National Key R\&D Program of China under Grant No. 2018YFA0404401; the National Natural Science Foundation of China under Grants No. 11835001, NO. 11921006, NO. 12035001, and NO. 11975282; the State Key Laboratory of Nuclear Physics and Technology, Peking University under Grant NPT2020KFY13; the China Postdoctoral Science Foundation under Grant No. BX20200136 and 2020M682747; the Strategic Priority Research Program of Chinese Academy of Sciences under Grant No. XDB34000000; the Key Research Program of the Chinese Academy of Sciences under Grant No. XDPB15; and the CUSTIPEN (China-U.S. Theory Institute for Physics with Exotic Nuclei) funded by the U.S. Department of Energy, Office of Science 
under Grant No. de-sc0009971. The High-Performance Computing Platform of Peking University is acknowledged.

Data Availability Statement: All of the relevant data are available from the corresponding author upon reasonable request.

Conflicts of Interest: The authors declare no conflict of interest.

\section{References}

1. Tanihata, I.; Savajols, H.; Kanungo, R. Recent experimental progress in nuclear halo structure studies. Prog. Part. Nucl. Phys. 2013, 68, 215-313. [CrossRef]

2. Motobayashi, T. World new facilities for radioactive isotope beams. EPJ Web Conf. 2014, 66, 01013. [CrossRef]

3. Blank, B.; Borge, M. Nuclear structure at the proton drip line: Advances with nuclear decay studies. Prog. Part. Nucl. Phys. 2008, 60, 403-483. [CrossRef]

4. Pfützner, M.; Karny, M.; Grigorenko, L.V.; Riisager, K. Radioactive decays at limits of nuclear stability. Rev. Mod. Phys. 2012, 84, 567-619. [CrossRef]

5. Michel, N.; Nazarewicz, W.; Płoszajczak, M.; Vertse, T. Shell model in the complex energy plane. J. Phys. G Nucl. Part. Phys. 2009, 36, 013101. [CrossRef]

6. Riisager, K. Nuclear halo states. Rev. Mod. Phys. 1994, 66, 1105-1116. [CrossRef]

7. Al-Khalili, J. An introduction to halo nuclei. In The Euroschool Lectures on Physics with Exotic Beams, Vol. I; Al-Khalili, J., Roeckl, E., Eds.; Springer: Berlin/Heidelberg, Germany, 2004; pp. 77-112. [CrossRef]

8. Kondo, Y.; Nakamura, T.; Tanaka, R.; Minakata, R.; Ogoshi, S.; Orr, N.A.; Achouri, N.L.; Aumann, T.; Baba, H.; Delaunay, F.; et al. Nucleus ${ }^{26}$ O: A barely unbound system beyond the drip Line. Phys. Rev. Lett. 2016, 116, 102503. [CrossRef]

9. Michel, N.; Nazarewicz, W.; Płoszajczak, M.; Okołowicz, J. Gamow shell model description of weakly bound nuclei and unbound nuclear states. Phys. Rev. C 2003, 67, 054311. [CrossRef]

10. Available online: https://www.nndc.bnl.gov/ensdf/ (accessed on 10 August 2021).

11. Renzi, F.; Raabe, R.; Randisi, G.; Smirnov, D.; Angulo, C.; Cabrera, J.; Casarejos, E.; Keutgen, T.; Ninane, A.; Charvet, J.L.; et al. Spectroscopy of ${ }^{7} \mathbf{H e}$ using the ${ }^{9} \mathbf{B e}\left({ }^{6} \mathbf{H e}_{,}^{8} \mathbf{B e}\right)$ transfer reaction. Phys. Rev. C 2016, 94, 024619. [CrossRef]

12. Votaw, D.; DeYoung, P.A.; Baumann, T.; Blake, A.; Boone, J.; Brown, J.; Chrisman, D.; Finck, J.E.; Frank, N.; Gombas, J.; et al. Low-lying level structure of the neutron-unbound $N=7$ isotones. Phys. Rev. C 2020, 102, 014325. [CrossRef]

13. Alkhazov, G.D.; Andronenko, M.N.; Dobrovolsky, A.V.; Egelhof, P.; Gavrilov, G.E.; Geissel, H.; Irnich, H.; Khanzadeev, A.V.; Korolev, G.A.; Lobodenko, A.A.; et al. Nuclear matter distributions in ${ }^{6} \mathrm{He}$ and ${ }^{8} \mathrm{He}$ from small angle p-He scattering in inverse kinematics at intermediate energy. Phys. Rev. Lett. 1997, 78, 2313-2316. [CrossRef]

14. Wang, L.B.; Mueller, P.; Bailey, K.; Drake, G.W.F.; Greene, J.P.; Henderson, D.; Holt, R.J.; Janssens, R.V.F.; Jiang, C.L.; Lu, Z.T.; et al. Laser spectroscopic determination of the ${ }^{6} \mathrm{He}$ nuclear charge radius. Phys. Rev. Lett. 2004, 93, 142501. [CrossRef]

15. Mueller, P.; Sulai, I.A.; Villari, A.C.C.; Alcántara-Núñez, J.A.; Alves-Condé, R.; Bailey, K.; Drake, G.W.F.; Dubois, M.; Eléon, C.; Gaubert, G.; et al. Nuclear charge radius of ${ }^{8}$ He. Phys. Rev. Lett. 2007, 99, 252501. [CrossRef]

16. Michel, N.; Nazarewicz, W.; Płoszajczak, M.; Rotureau, J. Antibound states and halo formation in the Gamow shell model. Phys. Rev. C 2006, 74, 054305. [CrossRef]

17. Papadimitriou, G.; Kruppa, A.T.; Michel, N.; Nazarewicz, W.; Płoszajczak, M.; Rotureau, J. Charge radii and neutron correlations in helium halo nuclei. Phys. Rev. C 2011, 84, 051304. [CrossRef]

18. Michel, N.; Li, J.G.; Xu, F.R.; Zuo, W. Two-neutron halo structure of ${ }^{31}$ F. Phys. Rev. C 2020, 101, 031301. [CrossRef]

19. Ma, Y.Z.; Xu, F.R.; Michel, N.; Zhang, S.; Li, J.G.; Hu, B.S.; Coraggio, L.; Itaco, N.; Gargano, A. Continuum and three-nucleon force in Borromean system: The ${ }^{17} \mathrm{Ne}$ case. Phys. Lett. B 2020, 808, 135673. [CrossRef]

20. Barrett, B.R.; Navrátil, P.; Vary, J.P. Ab initio no core shell model. Prog. Part. Nucl. Phys. 2013, 69, 131-181. [CrossRef]

21. Somà, V.; Cipollone, A.; Barbieri, C.; Navrátil, P.; Duguet, T. Chiral two- and three-nucleon forces along medium-mass isotope chains. Phys. Rev. C 2014, 89, 061301. [CrossRef]

22. Jansen, G.R.; Engel, J.; Hagen, G.; Navratil, P.; Signoracci, A. Ab initio coupled-cluster effective interactions for the shell model: Application to neutron-rich oxygen and carbon isotopes. Phys. Rev. Lett. 2014, 113, 142502. [CrossRef]

23. Hagen, G.; Hjorth-Jensen, M.; Jansen, G.; Papenbrock, T. Emergent properties of nuclei from ab initio coupled-cluster calculations. Phys.Scr. 2016, 91, 063006. [CrossRef]

24. Stroberg, S.R.; Calci, A.; Hergert, H.; Holt, J.D.; Bogner, S.K.; Roth, R.; Schwenk, A. Nucleus-dependent valence-space approach to nuclear structure. Phys. Rev. Lett. 2017, 118, 032502. [CrossRef]

25. Caurier, E.; Martínez-Pinedo, G.; Nowacki, F.; Poves, A.; Zuker, A.P. The shell model as a unified view of nuclear structure. Rev. Mod. Phys. 2005, 77, 427-488. [CrossRef]

26. Otsuka, T.; Gade, A.; Sorlin, O.; Suzuki, T.; Utsuno, Y. Evolution of shell structure in exotic nuclei. Rev. Mod. Phys. 2020, 92, 015002. [CrossRef]

27. Papadimitriou, G.; Rotureau, J.; Michel, N.; Płoszajczak, M.; Barrett, B.R. Ab initio no-core Gamow shell model calculations with realistic interactions. Phys. Rev. C 2013, 88, 044318. [CrossRef] 
28. Li, J.G.; Michel, N.; Hu, B.S.; Zuo, W.; Xu, F.R. Ab initio no-core Gamow shell-model calculations of multineutron systems. Phys. Rev. C 2019, 100, 054313. [CrossRef]

29. Li, J.G.; Michel, N.; Zuo, W.; Xu, F.R. Resonances of $A=4 T=1$ isospin triplet states within the ab initio no-core Gamow shell model. Phys. Rev. C 2021, 104, 024319. [CrossRef]

30. Navrátil, P.; Quaglioni, S.; Hupin, G.; Romero-Redondo, C.; Calci, A. Unified ab initio approaches to nuclear structure and reactions. Phys. Scr. 2016, 91, 053002. [CrossRef]

31. Hagen, G.; Hjorth-Jensen, M.; Jansen, G.R.; Machleidt, R.; Papenbrock, T. Continuum Effects and Three-Nucleon Forces in Neutron-Rich Oxygen Isotopes. Phys. Rev. Lett. 2012, 108, 242501. [CrossRef]

32. Hagen, G.; Hjorth-Jensen, M.; Jansen, G.R.; Machleidt, R.; Papenbrock, T. Evolution of Shell Structure in Neutron-Rich Calcium Isotopes. Phys. Rev. Lett. 2012, 109, 032502. [CrossRef]

33. Hu, B.S.; Wu, Q.; Sun, Z.H.; Xu, F.R. Ab initio Gamow in-medium similarity renormalization group with resonance and continuum. Phys. Rev. C 2019, 99, 061302. [CrossRef]

34. Volya, A.; Zelevinsky, V. Discrete and Continuum Spectra in the Unified Shell Model Approach. Phys. Rev. Lett. 2005, 94, 052501. [CrossRef]

35. Rotureau, J.; Okołowicz, J.; Płoszajczak, M. Microscopic theory of the two-proton radioactivity. Phys. Rev. Lett. 2005, 95, 042503. [CrossRef]

36. Id Betan, R.; Liotta, R.J.; Sandulescu, N.; Vertse, T. Two-particle resonant states in a many-body mean field. Phys. Rev. Lett. 2002, 89, 042501. [CrossRef]

37. Michel, N.; Nazarewicz, W.; Płoszajczak, M.; Bennaceur, K. Gamow shell model description of neutron-rich nuclei. Phys. Rev. Lett. 2002, 89, 042502. [CrossRef]

38. Sun, Z.H.; Wu, Q.; Zhao, Z.H.; Hu, B.S.; Dai, S.J.; Xu, F.R. Resonance and continuum Gamow shell model with realistic nuclear forces. Phys. Lett. B 2017, 769, 227-232. [CrossRef]

39. Ma, Y.Z.; Xu, F.R.; Coraggio, L.; Hu, B.S.; Li, J.G.; Fukui, T.; De Angelis, L.; Itaco, N.; Gargano, A. Chiral three-nucleon force and continuum for dripline nuclei and beyond. Phys. Lett. B 2020,802, 135257. [CrossRef]

40. Berggren, T. On the use of resonant states in eigenfunction expansions of scattering and reaction amplitudes. Nucl. Phys. A 1968, 109, 265-287. [CrossRef]

41. Hu, B.S.; Wu, Q.; Li, J.G.; Ma, Y.Z.; Sun, Z.H.; Michel, N.; Xu, F.R. An ab initio Gamow shell model approach with a core. Phys. Lett. B 2020, 802, 135206. [CrossRef]

42. Li, J.G.; Michel, N.; Zuo, W.; Xu, F.R. Unbound spectra of neutron-rich oxygen isotopes predicted by the Gamow shell model. Phys. Rev. C 2021, 103, 034305. [CrossRef]

43. Jaganathen, Y.; Id Betan, R.M.; Michel, N.; Nazarewicz, W.; Płoszajczak, M. Quantified Gamow shell model interaction for psd-shell nuclei. Phys. Rev. C 2017, 96, 054316. [CrossRef]

44. Hagen, G.; Hjorth-Jensen, M.; Michel, N. Gamow shell model and realistic nucleon-nucleon interactions. Phys. Rev. C 2006, 73, 064307. [CrossRef]

45. Tsukiyama, K.; Hjorth-Jensen, M.; Hagen, G. Gamow shell-model calculations of drip-line oxygen isotopes. Phys. Rev. C 2009, 80, 051301. [CrossRef]

46. Furnstahl, R.J.; Hagen, G.; Papenbrock, T. Corrections to nuclear energies and radii in finite oscillator spaces. Phys. Rev. C 2012, 86, 031301. [CrossRef]

47. Li, J.G.; Hu, B.S.; Wu, Q.; Gao, Y.; Dai, S.J.; Xu, F.R. Neutron-rich calcium isotopes within realistic Gamow shell model calculations with continuum coupling. Phys. Rev. C 2020, 102, 034302. [CrossRef]

48. Michel, N.; Li, J.G.; Xu, F.R.; Zuo, W. Proton decays in ${ }^{16} \mathrm{Ne}$ and ${ }^{18} \mathrm{Mg}$ and isospin-symmetry breaking in carbon isotopes and isotones. Phys. Rev. C 2021, 103, 044319. [CrossRef]

49. Michel, N.; Płoszajczak, M. The Gamow Shell Model: The Unified Theory of Nuclear Structure and Reactions; Springer: Berlin/Heidelberg, Germany, 2021. [CrossRef]

50. Dudek, J.; Szymański, Z.; Werner, T. Woods-Saxon potential parameters optimized to the high spin spectra in the lead region. Phys. Rev. C 1981, 23, 920-925. [CrossRef]

51. Gyarmati, B.; Vertse, T. On the normalization of Gamow functions. Nucl. Phys. A 1971, 160, 523-528. [CrossRef]

52. Newton, R. Scattering Theory of Waves and Particles; Dover Publications; New York, NY, USA, 2013. [CrossRef]

53. Michel, N.; Aktulga, H.; Jaganathen, Y. Toward scalable many-body calculations for nuclear open quantum systems using the Gamow Shell Model. Comp. Phys. Comm. 2020, 247, 106978. [CrossRef]

54. Michel, N.; Li, J.G.; Xu, F.R.; Zuo, W. Description of proton-rich nuclei in the $A \approx 20$ region within the Gamow shell model. Phys. Rev. C 2019, 100, 064303. [CrossRef]

55. Machleidt, R. High-precision, charge-dependent Bonn nucleon-nucleon potential. Phys. Rev. C 2001, 63, 024001. [CrossRef]

56. Machleidt, R.; Entem, D. Chiral effective field theory and nuclear forces. Phys. Rep. 2011, 503, 1-75. [CrossRef]

57. Hjorth-Jensen, M.; Kuo, T.T.; Osnes, E. Realistic effective interactions for nuclear systems. Phys. Rep. 1995, 261, 125-270. [CrossRef]

58. Coraggio, L.; Covello, A.; Gargano, A.; Itaco, N.; Kuo, T. Effective shell-model hamiltonians from realistic nucleon-nucleon potentials within a perturbative approach. Ann. Phys. 2012, 327, 2125-2151. [CrossRef] 
59. Bogner, S.; Furnstahl, R.; Schwenk, A. From low-momentum interactions to nuclear structure. Prog. Part. Nucl. Phys. 2010, 65, 94-147. [CrossRef]

60. Moshinsky, M. Transformation brackets for harmonic oscillator functions. Nucl. Phys. 1959, 13, 104-116. [CrossRef]

61. Kuo, T.; Lee, S.; Ratcliff, K. A folded-diagram expansion of the model-space effective hamiltonian. Nucl. Phys. A 1971, 176, 65-88. [CrossRef]

62. Suzuki, K.; Kumagai, H.; Okamoto, R.; Matsuzaki, M. Recursion method for deriving an energy-independent effective interaction. Phys. Rev. C 2014, 89, 044003. [CrossRef]

63. Takayanagi, K. Effective Hamiltonian in the extended Krenciglowa-Kuo method. Nucl. Phys. A 2011, 864, 91-112. [CrossRef]

64. Suzuki, Y.; Ikeda, K. Cluster-orbital shell model and its application to the He isotopes. Phys. Rev. C 1988, 38, 410-413. [CrossRef]

65. Thompson, D.; Lemere, M.; Tang, Y. Systematic investigation of scattering problems with the resonating-group method. Nucl. Phys. A 1977, 286, 53-66. [CrossRef]

66. Furutani, H.; Horiuchi, H.; Tamagaki, R. Cluster-model study of the $\mathrm{T}=1$ states in $\mathrm{A}=4$ system: $3 \mathrm{He}+\mathrm{p}$ scattering. Prog. Theor. Phys. 1979, 62, 981-1001. [CrossRef]

67. Furutani, H.; Kanada, H.; Kaneko, T.; Nagata, S.; Nishioka, H.; Okabe, S.; Saito, S.; Sakuda, T.; Seya, M. Study of non-alpha-nuclei based on the viewpoint of cluster correlations. Prog. Theor. Phys. Supp. 1980, 68, 193-302. [CrossRef]

68. Brown, B.A.; Richter, W.A. New “USD” Hamiltonians for the sd shell. Phys. Rev. C 2006, 74, 034315. [CrossRef]

69. Bogner, S.K.; Hergert, H.; Holt, J.D.; Schwenk, A.; Binder, S.; Calci, A.; Langhammer, J.; Roth, R. Nonperturbative shell-model interactions from the in-medium similarity renormalization group. Phys. Rev. Lett. 2014, 113, 142501. [CrossRef]

70. Becheva, E.; Blumenfeld, Y.; Khan, E.; Beaumel, D.; Daugas, J.M.; Delaunay, F.; Demonchy, C.E.; Drouart, A.; Fallot, M.; Gillibert, A.; et al. $N=14$ shell closure in ${ }^{22} \mathrm{O}$ viewed through a neutron sensitive probe. Phys. Rev. Lett. 2006, 96, 012501. [CrossRef]

71. Stanoiu, M.; Azaiez, F.; Dombrádi, Z.; Sorlin, O.; Brown, B.A.; Belleguic, M.; Sohler, D.; Saint Laurent, M.G.; Lopez-Jimenez, M.J.; Penionzhkevich, Y.E.; et al $N=14$ and 16 shell gaps in neutron-rich oxygen isotopes. Phys. Rev. C 2004, 69, 034312. [CrossRef]

72. Hoffman, C.R.; Baumann, T.; Bazin, D.; Brown, J.; Christian, G.; DeYoung, P.A.; Finck, J.E.; Frank, N.; Hinnefeld, J.; Howes, R.; et al Determination of the $N=16$ shell closure at the oxygen drip line. Phys. Rev. Lett. 2008, 100, 152502. [CrossRef]

73. Tshoo, K.; Satou, Y.; Bhang, H.; Choi, S.; Nakamura, T.; Kondo, Y.; Deguchi, S.; Kawada, Y.; Kobayashi, N.; Nakayama, Y.; et al. $N=16$ spherical shell closure in ${ }^{24}$ O. Phys. Rev. Lett. 2012, 109, 022501. [CrossRef]

74. Kohley, Z.; Baumann, T.; Bazin, D.; Christian, G.; DeYoung, P.A.; Finck, J.E.; Frank, N.; Jones, M.; Lunderberg, E.; Luther, B.; et al. Study of two-neutron radioactivity in the decay of ${ }^{26}$ O. Phys. Rev. Lett. 2013, 110, 152501. [CrossRef]

75. Ahn, D.S.; Fukuda, N.; Geissel, H.; Inabe, N.; Iwasa, N.; Kubo, T.; Kusaka, K.; Morrissey, D.J.; Murai, D.; Nakamura, T.; et al. Location of the neutron dripline at fluorine and neon. Phys. Rev. Lett. 2019, 123, 212501. [CrossRef]

76. Bagchi, S.; Kanungo, R.; Tanaka, Y.K.; Geissel, H.; Doornenbal, P.; Horiuchi, W.; Hagen, G.; Suzuki, T.; Tsunoda, N.; Ahn, D.S.; et al. Two-neutron halo is unveiled in ${ }^{29}$ F. Phys. Rev. Lett. 2020, 124, 222504. [CrossRef] [PubMed]

77. Revel, A.; Sorlin, O.; Marqués, F.M.; Kondo, Y.; Kahlbow, J.; Nakamura, T.; Orr, N.A.; Nowacki, F.; Tostevin, J.A.; Yuan, C.X.; et al. Extending the southern shore of the island of inversion to ${ }^{28} \mathrm{~F}$. Phys. Rev. Lett. 2020, 124, 152502. [CrossRef] [PubMed]

78. Wang, M.; Audi, G.; Kondev, F.; Huang, W.; Naimi, S.; Xu, X. The AME2016 atomic mass evaluation (II). Tables, graphs and references. Chin. Phys. C 2017, 41, 030003. [CrossRef]

79. Ekström, A.; Baardsen, G.; Forssén, C.; Hagen, G.; Hjorth-Jensen, M.; Jansen, G.R.; Machleidt, R.; Nazarewicz, W.; Papenbrock, T.; Sarich, J.; et al. Optimized chiral nucleon-nucleon interaction at next-to-next-to-leading order. Phys. Rev. Lett. 2013, 110, 192502. [CrossRef] [PubMed]

80. Fossez, K.; Rotureau, J.; Michel, N.; Nazarewicz, W. Continuum effects in neutron-drip-line oxygen isotopes. Phys. Rev. C 2017, 96, 024308. [CrossRef]

81. Stroberg, S.R.; Hergert, H.; Holt, J.D.; Bogner, S.K.; Schwenk, A. Ground and excited states of doubly open-shell nuclei from ab initio valence-space Hamiltonians. Phys. Rev. C 2016, 93, 051301. [CrossRef]

82. Otsuka, T.; Suzuki, T.; Holt, J.D.; Schwenk, A.; Akaishi, Y. Three-body forces and the limit of oxygen isotopes. Phys. Rev. Lett. 2010, 105, 032501. [CrossRef]

83. Utsuno, Y.; Otsuka, T.; Mizusaki, T.; Honma, M. Varying shell gap and deformation in $N \sim 20$ unstable nuclei studied by the Monte Carlo shell model. Phys. Rev. C 1999, 60, 054315. [CrossRef]

84. Stroberg, S.R.; Holt, J.D.; Schwenk, A.; Simonis, J. Ab initio limits of atomic nuclei. Phys. Rev. Lett. 2021, 126, 022501. [CrossRef]

85. Hammer, H.W.; Nogga, A.; Schwenk, A. Colloquium: Three-body forces: From cold atoms to nuclei. Rev. Mod. Phys. 2013, 85, 197-217. [CrossRef]

86. Hammer, H.W.; König, S.; van Kolck, U. Nuclear effective field theory: Status and perspectives. Rev. Mod. Phys. 2020, 92, 025004. [CrossRef]

87. Grigorenko, L.V.; Mukha, I.G.; Scheidenberger, C.; Zhukov, M.V. Two-neutron radioactivity and four-nucleon emission from exotic nuclei. Phys. Rev. C 2011, 84, 021303. [CrossRef]

88. Wu, Q.; Xu, F.R.; Hu, B.S.; Li, J.G. Perturbation calculations of nucleon-nucleon effective interactions in the Hartree-Fock basis. J. Phys. G Nucl. Part. Phys. 2019, 46, 055104. [CrossRef]

89. Tsunoda, N.; Otsuka, T.; Takayanagi, K.; Shimizu, N.; Suzuki, T.; Utsuno, Y.; Yoshida, S.; Ueno, H. The impact of nuclear shape on the emergence of the neutron dripline. Nature 2020, 587, 66-71. [CrossRef] [PubMed] 
90. Wienholtz, F.; Beck, D.; Blaum, K.; Borgmann, C.; Breitenfeldt, M.; Cakirli, R.B.; George, S.; Herfurth, F.; Holt, J.; Kowalska, M.; et al. Masses of exotic calcium isotopes pin down nuclear forces. Nature 2013, 498, 346. [CrossRef] [PubMed]

91. Steppenbeck, D.; Takeuchi, S.; Aoi, N.; Doornenbal, P.; Matsushita, M.; Wang, H.; Baba, H.; Fukuda, N.; Go, S.; Honma, M.; et al. Evidence for a new nuclear 'magic number'from the level structure of 54 Ca. Nature 2013, 502, 207. [CrossRef]

92. Michimasa, S.; Kobayashi, M.; Kiyokawa, Y.; Ota, S.; Ahn, D.S.; Baba, H.; Berg, G.P.A.; Dozono, M.; Fukuda, N.; Furuno, T.; et al. Magic nature of neutrons in ${ }^{54} \mathrm{Ca}$ : First mass measurements of ${ }^{55-57} \mathrm{Ca}$. Phys. Rev. Lett. 2018, 121, 022506. [CrossRef]

93. Riley, L.A.; McPherson, D.M.; Agiorgousis, M.L.; Baugher, T.R.; Bazin, D.; Bowry, M.; Cottle, P.D.; DeVone, F.G.; Gade, A.; Glowacki, M.T.; et al. Octupole strength in the neutron-rich calcium isotopes. Phys. Rev. C 2016, 93, 044327. [CrossRef]

94. Hergert, H.; Bogner, S.K.; Morris, T.D.; Binder, S.; Calci, A.; Langhammer, J.; Roth, R. Ab initio multireference in-medium similarity renormalization group calculations of even calcium and nickel isotopes. Phys. Rev. C 2014, 90, 041302. [CrossRef]

95. Holt, J.D.; Menéndez, J.; Simonis, J.; Schwenk, A. Three-nucleon forces and spectroscopy of neutron-rich calcium isotopes. Phys. Rev. C 2014, 90, 024312. [CrossRef]

96. Coraggio, L.; Covello, A.; Gargano, A.; Itaco, N. Realistic shell-model calculations for isotopic chains "north-east" of ${ }^{48}$ Ca in the $(\mathrm{N}, \mathrm{Z})$ plane. Phys. Rev. C 2014, 89, 024319. [CrossRef]

97. Neufcourt, L.; Cao, Y.; Nazarewicz, W.; Olsen, E.; Viens, F. Neutron drip line in the Ca region from Bayesian model averaging. Phys. Rev. Lett. 2019, 122, 062502. [CrossRef] [PubMed]

98. Coraggio, L.; De Gregorio, G.; Gargano, A.; Itaco, N.; Fukui, T.; Ma, Y.Z.; Xu, F.R. Shell-model study of calcium isotopes toward their drip line. Phys. Rev. C 2020, 102, 054326. [CrossRef]

99. Tarasov, O.B.; Ahn, D.S.; Bazin, D.; Fukuda, N.; Gade, A.; Hausmann, M.; Inabe, N.; Ishikawa, S.; Iwasa, N.; Kawata, K.; et al. Discovery of ${ }^{60} \mathrm{Ca}$ and implications for the stability of ${ }^{70} \mathrm{Ca}$. Phys. Rev. Lett. 2018, 121, 022501. [CrossRef]

100. Woodward, C.J.; Tribble, R.E.; Tanner, D.M. Mass of ${ }^{16}$ Ne. Phys. Rev. C 1983, 27, 27-30. [CrossRef]

101. KeKelis, G.J.; Zisman, M.S.; Scott, D.K.; Jahn, R.; Vieira, D.J.; Cerny, J.; Ajzenberg-Selove, F. Masses of the unbound nuclei ${ }^{16}$ Ne, ${ }^{15} \mathrm{~F}$, and ${ }^{12} \mathrm{O}$. Phys. Rev. C 1978, 17, 1929-1938. [CrossRef]

102. Brown, K.W.; Charity, R.J.; Sobotka, L.G.; Chajecki, Z.; Grigorenko, L.V.; Egorova, I.A.; Parfenova, Y.L.; Zhukov, M.V.; Bedoor, S.; Buhro, W.W.; et al. Observation of long-range three-body coulomb effects in the decay of ${ }^{16}$ Ne. Phys. Rev. Lett. 2014, $113,232501$. [CrossRef]

103. Li, J.G.; Michel, N.; Zuo, W.; Xu, F.R. Reexamining the variational two-particle reduced density matrix for nuclear systems. Phys. Rev. C 2021, 103, 064324. [CrossRef]

104. Aksyutina, Y.; Johansson, H.; Adrich, P.; Aksouh, F.; Aumann, T.; Boretzky, K.; Borge, M.; Chatillon, A.; Chulkov, L.; Cortina-Gil, D.; et al. Lithium isotopes beyond the drip line. Phys. Lett. B 2008, 666, 430-434. [CrossRef] 